Prepared in cooperation with the SOUTHWESTERN DIVISION NAVAL

FACILITIES ENGINEERING COMMAND

\title{
Diffusion Sampler Testing at Naval Air Station North Island, San Diego County, California, November 1999 to January 2000
}

Water-Resources Investigation 00-4182 


\section{Diffusion Sampler Testing at Naval Air Station North Island, San Diego County, California, November 1999 to January 2000}

\section{By DON A. VROBLESKY ${ }^{1}$ and BRIAN C. PETERS ${ }^{2}$}

U.S. Geological Survey

Water-Resources Investigation 00-4182

Prepared in cooperation with the

SOUTHWESTERN DIVISION NAVAL FACILITIES ENGINEERING COMMAND

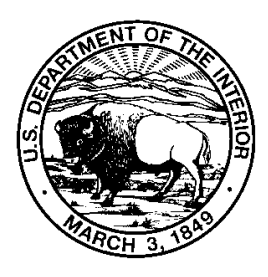

Columbia, South Carolina

2000

\footnotetext{
${ }^{1}$ U.S. Geological Survey, Columbia, South Carolina

${ }^{2} \mathrm{OHM}$ Remediation Services Corporation
} 


\title{
U.S. DEPARTMENT OF THE INTERIOR BRUCE BABBITT, Secretary
}

\author{
U.S. GEOLOGICAL SURVEY \\ Charles G. Groat, Director
}

The use of firm, trade, and brand names in this report is for identification purposes only and does not constitute endorsement by the U.S.

Geological Survey.

For additional information write to:

District Chief

U.S. Geological Survey

Stephenson Center - Suite 129

720 Gracern Road

Columbia, SC 29210-7651
Copies of this report can be purchased from:

U.S. Geological Survey

Branch of Information Center

Box 25286

Denver, CO 80225-0286 


\section{CONTENTS}

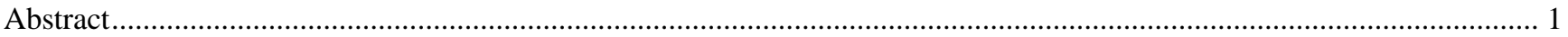

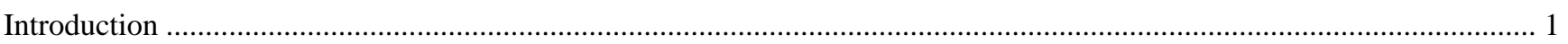

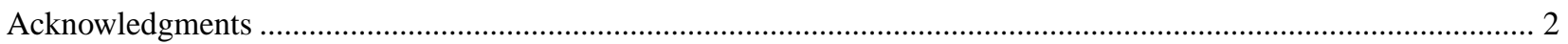

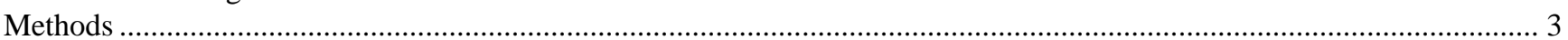

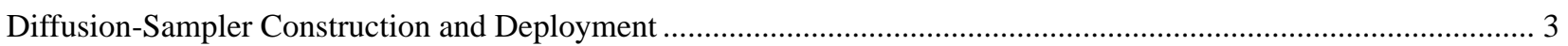

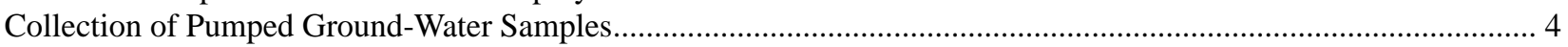

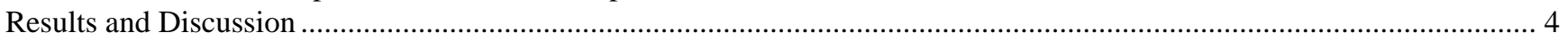

Comparison of Diffusion-Sampler Results to Bladder-Pump Results........................................................... 4

Comparison of Diffusion-Sampler Results to Peristaltic-Pump Results ......................................................... 16

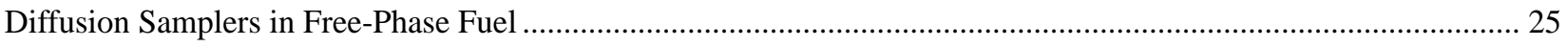

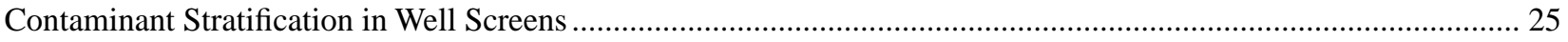

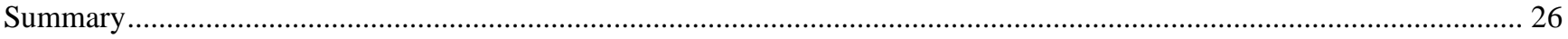

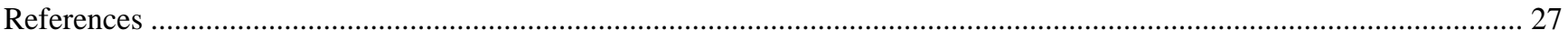

FIGURES

1. Map showing location of observation wells, Naval Air Station North Island, California ....................................... 2

2-9. Graphs showing:

2. Comparison of diffusion and low-flow samples in ground water at well MW-12,

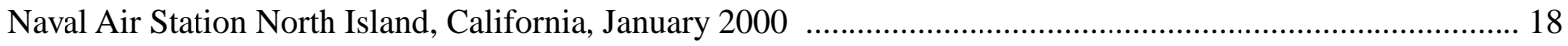

3. Comparison of diffusion and low-flow samples in ground water at well MW-13B,

Naval Air Station North Island, California, January 2000

4. Comparison of diffusion and low-flow samples in ground water at well PW-55,

Naval Air Station North Island, California, January 2000

5. Comparison of diffusion and low-flow samples in ground water at well MW-5, Naval

Air Station North Island, California, January 2000

6. Comparison of diffusion and low-flow samples in ground water at wells MW-68A,

MW-68B, MW-68C, and MW-68C2, and comparison of diffusion samples from

multiple wells to geologic log of well MW-68C, Naval Air Station North Island, California, January 2000 ..... 22

7. Comparison of trichloroethene concentrations in diffusion and low-flow samples in ground water at well PW-66, Naval Air Station North Island, California, January 2000

8. Comparison of diffusion and low-flow samples in ground water at well PW-15,

Naval Air Station North Island, California, January 2000

9. Comparison of diffusion and low-flow samples in ground water at well MW-13A,

Naval Air Station North Island, California, January 2000

\section{TABLES}

1. Summary of well information, Naval Air Station North Island, California

2. Sampler deployment and recovery information, Naval Air Station North Island, California, November 1999 to January 2000

3 Comparison of replicate samples collected by diffusion-sampler methodology, Naval Air

Station North Island, California, January 2000

4. Comparison of replicate samples collected by low-flow methodology, Naval Air Station

North Island, California, January 2000

5. Concentrations of selected chlorinated volatile organic compounds in water from diffusion

and low-flow sampling, Naval Air Station North Island, California, January 2000

6. Concentrations of benzene, ethylbenzene, toluene, and total xylenes in water from diffusion and low-flow sampling, Naval Air Station North Island, California, January 2000

7. Comparison of concentrations of selected volatile organic compounds in water from a diffusion sampler and in water from low-flow purging using a bladder pump at the same depth, Naval Air Station North Island, California, January 2000 .....

8. Concentrations of selected volatile organic compounds in free-phase jet fuel (JP-5) removed from ground water and in water from diffusion samplers deployed in a bucket containing the free-phase fuel, Naval Air Station North Island, California, January 2000 
CONVERSION FACTORS, VERTICAL DATUM, AND ABBREVIATIONS

\begin{tabular}{rcl}
\hline Multiply & By & To obtain \\
\hline & Length & \\
inch (in.) & 25.4 & millimeter \\
foot (ft) & 0.3048 & meter \\
mile (mi) & 1.609 & kilometer \\
& Area & \\
& 2.590 & square kilometer \\
square mile (mi ${ }^{2}$ ) & Flow & \\
& 0.3048 & meter per day \\
& 0.09294 & meter squared per day \\
foot per day (ft/d) & 0.06308 & liter per second \\
foot squared per day (ft $\left.{ }^{2} / \mathrm{d}\right)$ & 0.003785 & cubic meter per day \\
gallons per minute (gal/min) & millimeters per year \\
gallons per day (gal/d) & Volume & \\
inches per year (in/yr) & 3.4 & liter \\
& & \\
gallon (gal) & 3.785 & \\
& &
\end{tabular}

Temperature: In this report, temperature is given in degrees Celsius $\left({ }^{\circ} \mathrm{C}\right)$, which can be converted to degrees Fahrenheit $\left({ }^{\circ} \mathrm{F}\right)$ by the following equation:

$$
{ }^{\circ} \mathrm{F}=\left(9 / 5 \times \mathrm{C}^{\circ}\right)+32
$$

Sea Level: In this report, "sea level" refers to the National Geodetic Vertical Datum of 1929-a geodetic datum derived from a general adjustment of the first-order level nets of the United States and Canada, formerly called Sea Level Datum of 1929.

Chemical concentration: In this report, chemical concentration in water is expressed in metric units as milligrams per liter (mg/L) or micrograms per liter $(\mu \mathrm{g} / \mathrm{L})$.

\section{Other units used in report:}

$\begin{array}{ll}\text { cubic feet per day } & \mathrm{ft}^{3} / \mathrm{d} \\ \text { cubic feet per milligram } & \mathrm{ft}^{3} / \mathrm{mg} \\ \text { degrees Celsius } & { }^{\circ} \mathrm{C} \\ \text { gram } & \mathrm{g} \\ \text { liter } & \mathrm{L} \\ \text { microgram } & \mu \mathrm{g} \\ \text { micrometer } & \mu \mathrm{m} \\ \text { microliter } & \mu \mathrm{L} \\ \text { milligram } & \mathrm{mg} \\ \text { milliliter } & \mathrm{mL} \\ \text { milliliter per minute } & \mathrm{mL} / \mathrm{min}\end{array}$

\footnotetext{
Abbreviations used in this report:

bls - below land surface

11DCA - 1,1-dichloroethane

11DCE - 1,1-dichloroethene

$c \mathrm{DCE}$ - cis-1,2-dichloroethene

MCL - maximum contaminant level

PCE - tetrachloroethene

PVC - polyvinyl chloride

TCA - 1,1,1-trichloroethane

TCE - trichloroethene

USEPA - U.S. Environmental Protection Agency

USGS - U.S. Geological Survey
}

iv Diffusion Sampler Testing at Naval Air Station North Island, San Diego County, California, November 1999 to January 2000 


\title{
Diffusion Sampler Testing at Naval Air Station North Island, San Diego County, California, November 1999 to January 2000
}

\author{
By Don A. Vroblesky and Brian C. Peters
}

ABSTRACT

Volatile organic compound concentrations in water from diffusion samplers were compared to concentrations in water obtained by low-flow purging at 15 observation wells at the Naval Air Station North Island, San Diego, California. Multiple diffusion samplers were installed in the wells. In general, comparisons using bladder pumps and diffusion samplers showed similar volatile organic carbon concentrations. In some wells, sharp concentration gradients were observed, such as an increase in cis-1,2-dichloroethene concentration from 100 to 2,600 micrograms per liter over a vertical distance of only 3.4 feet. In areas where such sharp gradients were observed, concentrations in water obtained by low-flow sampling at times reflected an average concentration over the area of influence; however, concentrations obtained by using the diffusion sampler seemed to represent the immediate vicinity of the sampler. When peristaltic pumps were used to collect ground-water samples by low-flow purging, the volatile organic compound concentrations commonly were lower than concentrations obtained by using diffusion samplers. This difference may be due to loss of volatiles by degassing under negative pressures in the sampling lines induced while using the peristaltic pump, mixing in the well screen, or possible short-circuiting of water from an adjacent depth. Diffusion samplers placed in buckets of freephase jet fuel (JP-5) and Stoddard solvent from observation wells did not show evidence of structural integrity loss during the 2 months of equilibration, and volatile organic compounds detected in the free-phase fuel also were detected in the water from the diffusion samplers.

\section{INTRODUCTION}

Low-density polyethylene diffusion samplers, filled with deionized water or air, have been shown to be an inexpensive alternative sampling method for volatile organic compounds (VOCs) in contaminated wells or in ground-water discharge zones beneath surface-water bodies (Vroblesky and others, 1996; Vroblesky and Robertson, 1996; Vroblesky and Hyde, 1997; Vroblesky and others, 1999; Gefell and others, 1999). The use of diffusion samplers in wells has generated substantial interest due to their capability to sample ground water without the need for prior well purging.

The Naval Air Station (NAS) North Island, in San Diego County, California, has been used since 1917 as an air station, harbor, and training base. The base is approximately 1 mile west across San Diego Bay from the San Diego metropolitan area (fig. 1). Activities at the base have resulted in ground-water contamination by a variety of compounds, including chlorinated aliphatic hydrocarbons and petroleum hydrocarbons. In addition, free-phase JP-5 jet fuel and Stoddard solvent (mineral spirits) are present locally in the ground water. Stoddard solvent is a refined petroleum product typically used as a thinning agent for paints, coatings, waxes, printing inks, and adhesives; a solvent in photocopy toners and in dry cleaning; and as a degreaser for engine parts.

The purpose of this report is to present the findings of an investigation to determine whether the use of polyethylene deionized-water-filled diffusion 


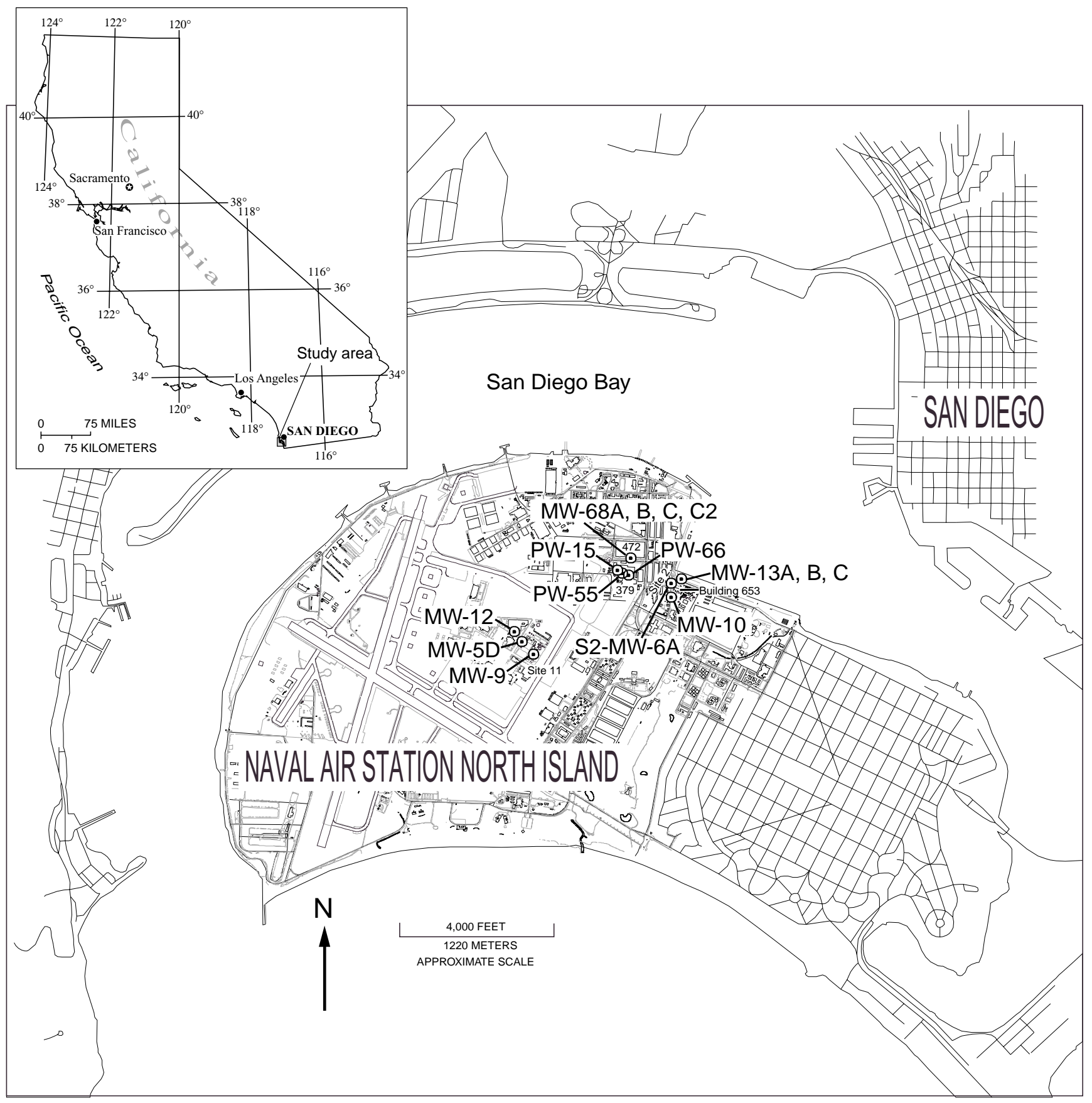

Figure 1. Location of observation wells, Naval Air Station North Island, California.

samplers is a viable method of sampling VOCs in ground water at the base. VOC concentrations in water obtained from diffusion samplers set at multiple levels in wells are compared to VOC concentrations in water obtained from low-flow sampling. Diffusion samplers were placed in 15 observation wells, and 2 samplers were placed in buckets of free-phase JP-5 and Stoddard solvent.

\section{Acknowledgments}

The Department of the Navy, Southwestern Division Naval Facilities Command, funded this work. The fieldwork was a cooperative effort between the U.S. Geological Survey and OHM Remediation Services Corp. 


\section{METHODS}

Diffusion samplers were tested in 15 wells at NAS North Island, California. VOC concentrations in water from the diffusion samplers were compared to VOC concentrations in water from low-flow sampling ports open adjacent to each diffusion sampler. Lowflow sampling was accomplished by using a peristaltic pump at most sites and a bladder pump at selected sites.

\section{Diffusion-Sampler Construction and Deployment}

Each diffusion sampler consisted of a 2-inchdiameter, low-density polyethylene (LDPE) tube heatsealed at both ends and containing deionized water. On the outside of each sampler, an LDPE mesh provided abrasion protection. This sampling methodology is patented (patent number 5,804,743) and is available for non-exclusive licensing from the U.S. Geological Survey Technology Enterprise Office, Mail Stop 211, National Center, 12201 Sunrise Valley Drive, Reston, Virginia (telephone 703 648-4450; fax 703 648-4408).
Diffusion samplers were attached to intakes of bladder pumps by means of plastic cable ties. Attached to each remaining diffusion sampler was a Tygon tube extending from the sampler to land surface. The tubing was secured to the diffusion sampler and to a weighted line at approximately 10 -foot intervals by using plastic cable ties. The purpose of the tubing was to allow ground water to be collected adjacent to each diffusion sampler by using low-flow methodology with a peristaltic pump.

The diffusion samplers were deployed in 15 wells at NAS North Island during November 11, 1999 (table 1). All wells were constructed of 4-inchdiameter casing. The samplers were attached by plastic cable ties to either a weighted line or a 1/2-inch (outside diameter) PVC pipe. When multiple sections of PVC pipe were required to reach the top of the casing, the sections were joined using stainless-steel screws. The PVC pipe was secured to the top of the well casing to prevent the diffusion samplers from shifting during the equilibration period.

Two of the sampled wells (PW-15 and PW-55) contained floating nonaqueous-phase liquid (LNAPL) consisting of free-phase petroleum and Stoddard solvent. To install diffusion samplers in these wells, a

Table 1. Summary of well information, Naval Air Station North Island, California

[ft, feet; ft bls, feet below land surface; ft msl, feet relative to mean sea level; Elev., elevation; NM, not measured; NA, not available; TOC, top of casing; A, bladder pump attached to the diffusion sampler; B, peristaltic pump using tubing attached to individual diffusion samplers; $\mathrm{C}$, same as B, except one depth was sampled using a bladder pump attached to a diffusion sampler; D, same as $\mathrm{B}$, except the well was resampled using a bladder pump following removal of the diffusion samplers]

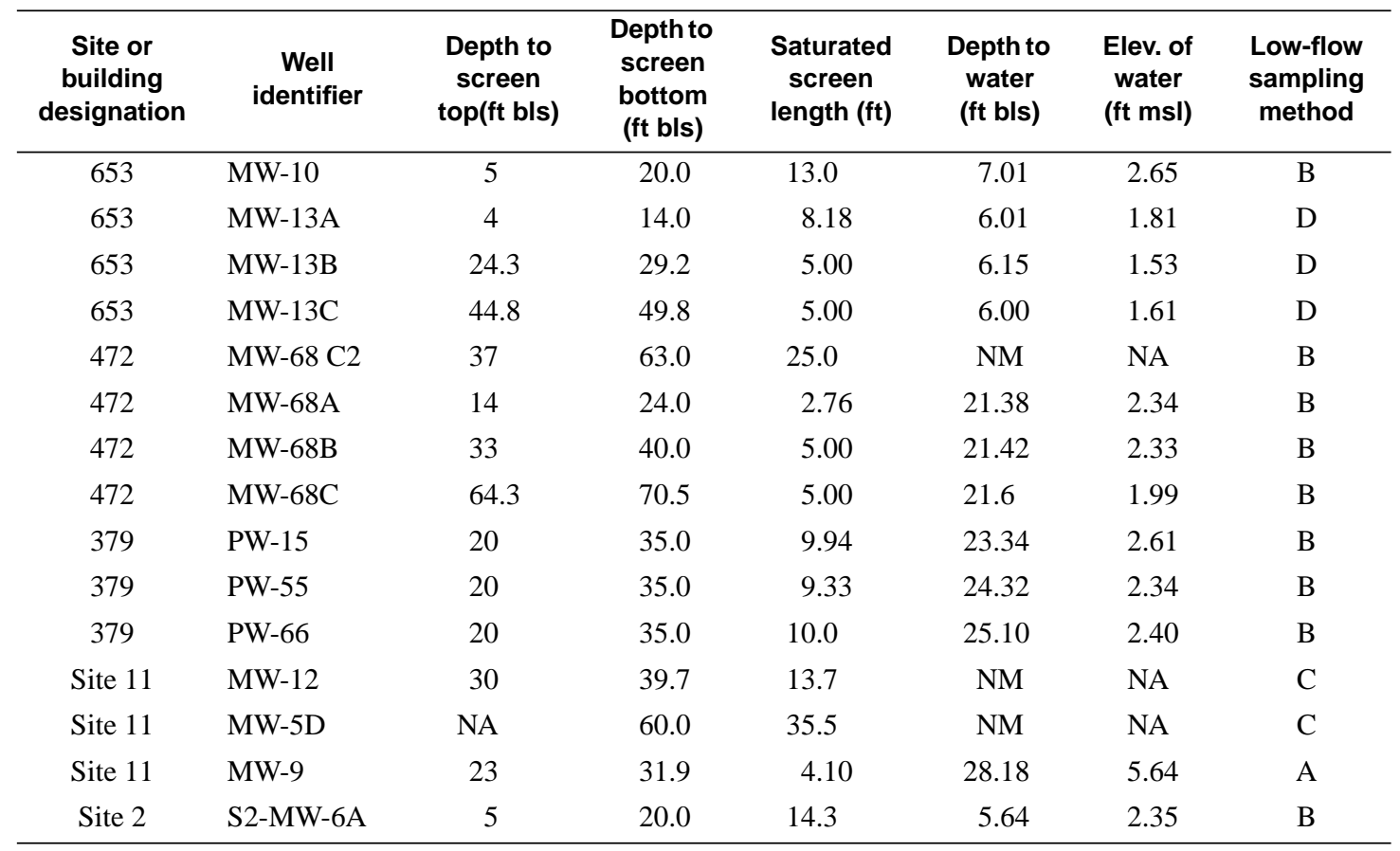


rubber cap was placed on the lower end of a section of 2-inch-diameter PVC pipe and lowered into the well to a depth below the LNAPL. The top end of the pipe extended to land surface. A smaller diameter pipe then was used to pound out the rubber cap, which was recovered from the well along the outside of the 2-inch-diameter pipe by means of a rope attached to the cap. The diffusion samplers were lowered into the well through the 2-inch-diameter pipe, thereby avoiding direct contact with the LNAPL. The pipe was secured in place to allow the diffusion sampler to be recovered without contact with the LNAPL.

\section{Collection of Pumped Ground-Water Samples}

The diffusion samplers were allowed to remain undisturbed in the well water for 65 to 71 days (table 2). The wells were sampled at the time of sampler recovery using low-flow techniques. Low-flow sampling consisted of purging the well by means of using a dedicated bladder pump or a peristaltic pump connected to the Tygon tubing that had been attached to each of the diffusion samplers prior to deployment. Purging was done at a rate of 120 milliliters per minute until measurements of $\mathrm{pH}$, water temperature, and specific conductance stabilized. In general, purging involved about 20 minutes of pumping and removal of less than 1 gallon of water from each sampling port. Decontamination of equipment was not required because each sampling interval had dedicated tubing.

A variety of methods were used to retrieve the diffusion samplers and to low-flow sample the well. The first method of sample retrieval involved recovering the diffusion sampler from the well immediately following low-flow sampling by using a bladder pump from the depth at which the diffusion sampler had equilibrated (well MW-9 only). A second method involved using a peristaltic pump to low-flow sample ground water adjacent to each of the diffusion samplers by means of the dedicated Tygon tubing attached to each diffusion sampler. The depths were low-flow sampled beginning with the shallowest and proceeding to the deepest. In some wells (MW-5 and MW-12), one of the depths was sampled using a dedicated bladder pump while the remaining depths were sampled using a bladder pump attached to dedicated tubing. Two wells (MW-13A and MW-13B) were low-flow sampled by using a peristaltic pump, the diffusion samplers were recovered, a bladder pump was inserted into each well, and the wells were then immediately resampled by low-flow methodology using the bladder pump.

The diffusion samplers were recovered from the wells by means of the attached weighted line or PVC pipe. The samplers were cut open, and the water was slowly decanted into glass vials pretreated with hydrochloric acid. The water samples were sent to a contract laboratory for analysis by using Environmental Protection Agency Method 8260B (U.S. Environmental Protection Agency, 1999). Replicate samples were collected from approximately 10 percent of the sampling sites. In general, both diffusion samples and low-flow samples compared well with their respective replicate samples (tables 3 and 4).

\section{RESULTS AND DISCUSSION}

VOC concentrations in water obtained from diffusion samplers were similar to concentrations obtained by using low-flow sampling methods for most of the tested wells (tables 5 and 6, respectively). As will be shown, most concentration differences between the two sampling methods probably can be attributed to VOC degassing during peristaltic-pump sampling or to in-well mixing.

\section{Comparison of Diffusion-Sampler Results to Bladder-Pump Results}

Tests showing the most direct comparison between diffusion sampling and low-flow sampling were in wells where a bladder pump was used to lowflow sample. The test producing the least amount of well-water disturbance was in well MW-9 where a diffusion sampler was recovered immediately following low-flow sampling using a bladder pump from the same depth. Concentrations of 1,1-dichloroethene (1,1-DCE) and trichloroethene (TCE) obtained using the diffusion sampler agreed well (12 and 3 percent difference, respectively) with those obtained using the bladder pump (table 7). The difference is about the same as the differences (approximately 12 percent) in 1,1-DCE and TCE concentrations measured in replicate samples collected by using a dedicated bladder pump at well MW-5D (table 4). Thus, 12 percent is within the sample-collection variability for 1,1-DCE and TCE. Agreement between the methods was poorer for tetrachloroethene (PCE) concentrations. The PCE concentration in water from the diffusion sampler was 21 percent lower than the concentration in water 
Table 2. Sampler deployment and recovery information, Naval Air Station North Island, California, November 1999 to January 2000

[repl, replicate sample; NA, not applicable; *, low-flow bladder-pump sample; \#, data from OHM Remediation Services Corporation (2000)]

\begin{tabular}{|c|c|c|c|c|c|c|c|c|}
\hline $\begin{array}{c}\text { Site or } \\
\text { building } \\
\text { designation }\end{array}$ & $\begin{array}{c}\text { Well } \\
\text { identifier }\end{array}$ & $\begin{array}{c}\text { Sampling } \\
\text { interval } \\
\text { identifier }\end{array}$ & $\begin{array}{l}\text { Low-flow } \\
\text { sample } \\
\text { laboratory } \\
\text { identifier }\end{array}$ & $\begin{array}{l}\text { Diffusion- } \\
\text { sampler } \\
\text { laboratory } \\
\text { identifier }\end{array}$ & $\begin{array}{l}\text { Depth to } \\
\text { diffusion- } \\
\text { sampler } \\
\text { center } \\
\text { (ft bls) }\end{array}$ & $\begin{array}{c}\text { Date } \\
\text { installed }\end{array}$ & $\begin{array}{l}\text { Date } \\
\text { recovered }\end{array}$ & $\begin{array}{c}\text { Number of } \\
\text { days } \\
\text { diffusion } \\
\text { samplers } \\
\text { were in } \\
\text { wells }\end{array}$ \\
\hline 653 & MW-10 & $\mathrm{A}$ & $779679-0091$ & 779679-0099 & 7.75 & $11 / 12 / 99$ & $1 / 18 / 00$ & 67 \\
\hline 653 & MW-10 & B & 779679-0092 & 779679-0100 & 9.15 & $11 / 12 / 99$ & $1 / 18 / 00$ & 67 \\
\hline 653 & MW-10 & $\mathrm{C}$ & 779679-0093 & 779679-0101 & 11.1 & $11 / 12 / 99$ & $1 / 18 / 00$ & 67 \\
\hline 653 & MW-10 & $\mathrm{D}$ & 779679-0094 & 779679-0102 & 13.1 & $11 / 12 / 99$ & $1 / 18 / 00$ & 67 \\
\hline 653 & MW-10 & $\mathrm{E}$ & 779679-0095 & 779679-0103 & 15.1 & $11 / 12 / 99$ & $1 / 18 / 00$ & 67 \\
\hline 653 & MW-10 & $\mathrm{F}$ & 779679-0096 & 779679-0104 & 17.1 & $11 / 12 / 99$ & $1 / 18 / 00$ & 67 \\
\hline 653 & MW-10 & $\mathrm{G}$ & 779679-0097 & 779679-0105 & 18.8 & $11 / 12 / 99$ & $1 / 18 / 00$ & 67 \\
\hline 653 & MW-10 & G repl & 779679-0098 & NA & 18.8 & 11/12/99 & $1 / 18 / 00$ & 67 \\
\hline 653 & MW-13A & A & 779679-0030 & 779679-0042 & 6.50 & $11 / 10 / 99$ & $1 / 17 / 00$ & 68 \\
\hline 653 & MW-13A & B & 779679-0031 & 779679-0043 & 7.95 & 11/10/99 & $1 / 17 / 00$ & 68 \\
\hline 653 & MW-13A & $\mathrm{C}$ & 779679-0032 & 779679-0044 & 9.35 & $11 / 10 / 99$ & $1 / 17 / 00$ & 68 \\
\hline 653 & MW-13A & $\mathrm{D}$ & 779679-0033 & 779679-0045 & 10.9 & $11 / 10 / 99$ & $1 / 17 / 00$ & 68 \\
\hline 653 & MW-13A & $\mathrm{E}$ & 779679-0034 & 779679-0046 & 12.4 & 11/10/99 & $1 / 17 / 00$ & 68 \\
\hline 653 & MW-13A*\# & NA & NA & NA & NA & NA & $1 / 17 / 00$ & NA \\
\hline 653 & MW-13B & A & 779679-0035 & 779679-0047 & 24.8 & 11/10/99 & $1 / 17 / 00$ & 68 \\
\hline 653 & MW-13B & $\mathrm{B}$ & 779679-0036 & 779679-0048 & 26.1 & $11 / 10 / 99$ & $1 / 17 / 00$ & 68 \\
\hline 653 & MW-13B & $\mathrm{C}$ & 779679-0037 & 779679-0049 & 27.5 & $11 / 10 / 99$ & $1 / 17 / 00$ & 68 \\
\hline 653 & MW-13B*\# & NA & NA & NA & NA & NA & $1 / 17 / 00$ & NA \\
\hline 653 & MW-13C & $\mathrm{A}$ & 779679-0038 & 779679-0054 & 45.4 & $11 / 10 / 99$ & $1 / 17 / 00$ & 68 \\
\hline 653 & MW-13C & $\mathrm{B}$ & 779679-0039 & 779679-0055 & 46.6 & 11/10/99 & $1 / 17 / 00$ & 68 \\
\hline 653 & MW-13C & B repl & 779679-0041 & NA & 46.6 & 11/10/99 & $1 / 17 / 00$ & 68 \\
\hline 653 & MW-13C & $\mathrm{C}$ & 779679-0040 & 779679-0056 & 48.0 & 11/10/99 & $1 / 17 / 00$ & 68 \\
\hline 653 & MW-13C*\# & NA & NA & NA & NA & NA & $1 / 17 / 00$ & NA \\
\hline 472 & MW-68A & A & 779679-0023 & 779679-0025 & 21.7 & $11 / 9 / 99$ & $1 / 17 / 00$ & 69 \\
\hline 472 & MW-68A & B & 779679-0024 & 779679-0026 & 23.0 & $11 / 9 / 99$ & $1 / 17 / 00$ & 69 \\
\hline 472 & MW-68B & A & 779679-0016 & 779679-0020 & 34.5 & $11 / 9 / 99$ & $1 / 17 / 00$ & 69 \\
\hline 472 & MW-68B & B & 779679-0017 & 779679-0021 & 37.0 & $11 / 9 / 99$ & $1 / 17 / 00$ & 69 \\
\hline 472 & MW-68B & B repl & 779679-0018 & NA & 37.0 & $11 / 9 / 99$ & $1 / 17 / 00$ & 69 \\
\hline 472 & MW-68B & $\mathrm{C}$ & 779679-0019 & 779679-0022 & 38.5 & 11/9/99 & $1 / 17 / 00$ & 69 \\
\hline 472 & MW-68C & A & 779679-0027 & 779679-0050 & 56.0 & $11 / 9 / 99$ & $1 / 17 / 00$ & 69 \\
\hline 472 & MW-68C & B & 779679-0028 & 779679-0051 & 57.5 & $11 / 9 / 99$ & $1 / 17 / 00$ & 69 \\
\hline 472 & MW-68C & $\mathrm{C}$ & 779679-0117 & 779679-0116 & 59.0 & $11 / 9 / 99$ & $1 / 19 / 00$ & 71 \\
\hline
\end{tabular}


Table 2. Sampler deployment and recovery information, Naval Air Station North Island, California, November 1999 to January 2000-Continued

[repl, replicate sample; NA, not applicable; *, low-flow bladder-pump sample; \#, data from OHM Remediation Services Corporation (2000)]

\begin{tabular}{|c|c|c|c|c|c|c|c|c|}
\hline $\begin{array}{c}\text { Site or } \\
\text { building } \\
\text { designation }\end{array}$ & $\begin{array}{c}\text { Well } \\
\text { identifier }\end{array}$ & $\begin{array}{c}\text { Sampling } \\
\text { interval } \\
\text { identifier }\end{array}$ & $\begin{array}{l}\text { Low-flow } \\
\text { sample } \\
\text { laboratory } \\
\text { identifier }\end{array}$ & $\begin{array}{l}\text { Diffusion- } \\
\text { sampler } \\
\text { laboratory } \\
\text { identifier }\end{array}$ & $\begin{array}{l}\text { Depth to } \\
\text { diffusion- } \\
\text { sampler } \\
\text { center } \\
\text { (ft bls) }\end{array}$ & $\begin{array}{c}\text { Date } \\
\text { installed }\end{array}$ & $\begin{array}{l}\text { Date } \\
\text { recovered }\end{array}$ & $\begin{array}{c}\text { Number } \\
\text { of days } \\
\text { diffusion } \\
\text { samplers } \\
\text { were in } \\
\text { wells }\end{array}$ \\
\hline 472 & MW-68C2 & $\mathrm{A}$ & 779679-0166 & $779679-0181$ & 37.2 & $11 / 11 / 99$ & $1 / 20 / 00$ & 70 \\
\hline 472 & MW-68C2 & B & 779679-0169 & 779679-0182 & 39.1 & $11 / 11 / 99$ & $1 / 20 / 00$ & 70 \\
\hline 472 & MW-68C2 & B repl & NA & 779679-0183 & 39.1 & $11 / 11 / 99$ & $1 / 20 / 00$ & 70 \\
\hline 472 & MW-68C2 & $\mathrm{C}$ & 779679-0171 & 779679-0184 & 40.5 & $11 / 11 / 99$ & $1 / 20 / 00$ & 70 \\
\hline 472 & MW-68C2 & $\mathrm{D}$ & 779679-0173 & 779679-0185 & 42.0 & $11 / 11 / 99$ & $1 / 20 / 00$ & 70 \\
\hline 472 & MW-68C2 & $\mathrm{E}$ & 779679-0176 & 779679-0186 & 44.1 & $11 / 11 / 99$ & $1 / 20 / 00$ & 70 \\
\hline 472 & MW-68C2 & $\mathrm{F}$ & 779679-0178 & 779679-0187 & 46.0 & $11 / 11 / 99$ & $1 / 20 / 00$ & 70 \\
\hline 472 & MW-68C2 & $\mathrm{G}$ & 779679-0180 & 779679-0188 & 47.8 & $11 / 11 / 99$ & $1 / 20 / 00$ & 70 \\
\hline 472 & MW-68C2 & $\mathrm{H}$ & 779679-0179 & 779679-0189 & 49.8 & $11 / 11 / 99$ & $1 / 20 / 00$ & 70 \\
\hline 472 & MW-68C2 & I & 779679-0177 & 779679-0190 & 51.9 & $11 / 11 / 99$ & $1 / 20 / 00$ & 70 \\
\hline 472 & MW-68C2 & $\mathrm{J}$ & 779679-0174 & 779679-0191 & 53.9 & $11 / 11 / 99$ & $1 / 20 / 00$ & 70 \\
\hline 472 & MW-68C2 & $\mathrm{J}$ repl & 779679-0175 & NA & 53.9 & $11 / 11 / 99$ & $1 / 20 / 00$ & 70 \\
\hline 472 & MW-68C2 & $\mathrm{K}$ & 779679-0172 & 779679-0192 & 55.5 & $11 / 11 / 99$ & $1 / 20 / 00$ & 70 \\
\hline 472 & MW-68C2 & $\mathrm{L}$ & 779679-0170 & 779679-0193 & 57.5 & $11 / 11 / 99$ & $1 / 20 / 00$ & 70 \\
\hline 472 & MW-68C2 & M & 779679-0168 & 779679-0194 & 59.5 & $11 / 11 / 99$ & $1 / 20 / 00$ & 70 \\
\hline 472 & MW-68C2 & M repl & NA & 779679-0195 & 59.5 & $11 / 11 / 99$ & $1 / 20 / 00$ & 70 \\
\hline 472 & MW-68C2 & $\mathrm{N}$ & 779679-0167 & 779679-0196 & 61.5 & $11 / 11 / 99$ & $1 / 20 / 00$ & 70 \\
\hline 379 & PW-15 & A & $779679-0083$ & 779679-0089 & 25.4 & 11/13/99 & $1 / 19 / 00$ & 67 \\
\hline 379 & PW-15 & B & 779679-0084 & 779679-0118 & 27.1 & $11 / 13 / 99$ & $1 / 19 / 00$ & 67 \\
\hline 379 & PW-15 & B repl & NA & 779679-0119 & 27.1 & $11 / 13 / 99$ & $1 / 19 / 00$ & 67 \\
\hline 379 & PW-15 & $\mathrm{C}$ & 779679-0085 & 779679-0156 & 28.5 & 11/13/99 & $1 / 19 / 00$ & 67 \\
\hline 379 & PW-15 & $\mathrm{D}$ & 779679-0086 & 779679-0157 & 30.2 & $11 / 13 / 99$ & $1 / 19 / 00$ & 67 \\
\hline 379 & PW-15 & $\mathrm{E}$ & 779679-0087 & 779679-0158 & 31.7 & $11 / 13 / 99$ & $1 / 19 / 00$ & 67 \\
\hline 379 & PW-15 & E repl & NA & 779679-0159 & 31.7 & $11 / 13 / 99$ & $1 / 19 / 00$ & 67 \\
\hline 379 & PW-15 & $\mathrm{F}$ & 779679-0088 & 779679-0164 & 33.1 & 11/13/99 & $1 / 19 / 00$ & 67 \\
\hline 379 & PW-55 & A & 779679-0077 & 779679-0109 & 27.1 & $11 / 13 / 99$ & $1 / 19 / 00$ & 67 \\
\hline 379 & PW-55 & B & 779679-0078 & 779679-0110 & 28.9 & $11 / 13 / 99$ & $1 / 19 / 00$ & 67 \\
\hline 379 & PW-55 & B repl & NA & 779679-0114 & 28.9 & $11 / 13 / 99$ & $1 / 19 / 00$ & 67 \\
\hline 379 & PW-55 & $\mathrm{C}$ & 779679-0079 & 779679-0111 & 30.6 & $11 / 13 / 99$ & $1 / 19 / 00$ & 67 \\
\hline 379 & PW-55 & $\mathrm{D}$ & 779679-0080 & 779679-0112 & 31.9 & 11/13/99 & $1 / 19 / 00$ & 67 \\
\hline 379 & PW-55 & $\mathrm{E}$ & 779679-0081 & 779679-0113 & 33.1 & 11/13/99 & $1 / 19 / 00$ & 67 \\
\hline 379 & PW-55 & E repl & 779679-0082 & 779679-0115 & 33.1 & 11/13/99 & $1 / 19 / 00$ & 67 \\
\hline 379 & PW-66 & A & 779679-0106 & 779679-0145 & 25.5 & $11 / 10 / 99$ & $1 / 18 / 00$ & 69 \\
\hline 379 & PW-66 & $\mathrm{B}$ & 779679-0107 & 779679-0146 & 27.3 & $11 / 10 / 99$ & $1 / 18 / 00$ & 69 \\
\hline 379 & PW-66 & B repl & NA & 779679-0151 & 27.3 & 11/10/99 & $1 / 18 / 00$ & 69 \\
\hline
\end{tabular}


Table 2. Sampler deployment and recovery information, Naval Air Station North Island, California, November 1999 to January 2000-Continued

[repl, replicate sample; NA, not applicable; *, low-flow bladder-pump sample; \#, data from OHM Remediation Services Corporation (2000)]

\begin{tabular}{|c|c|c|c|c|c|c|c|c|}
\hline $\begin{array}{c}\text { Site or } \\
\text { building } \\
\text { designation }\end{array}$ & $\begin{array}{c}\text { Well } \\
\text { identifier }\end{array}$ & $\begin{array}{l}\text { Sampling } \\
\text { interval } \\
\text { identifier }\end{array}$ & $\begin{array}{l}\text { Low-flow } \\
\text { sample } \\
\text { laboratory } \\
\text { identifier }\end{array}$ & $\begin{array}{l}\text { Diffusion- } \\
\text { sampler } \\
\text { laboratory } \\
\text { identifier }\end{array}$ & $\begin{array}{l}\text { Depth to } \\
\text { diffusion- } \\
\text { sampler } \\
\text { center } \\
\text { (ft bls) }\end{array}$ & $\begin{array}{c}\text { Date } \\
\text { installed }\end{array}$ & $\begin{array}{l}\text { Date } \\
\text { recovered }\end{array}$ & $\begin{array}{c}\text { Number } \\
\text { of days } \\
\text { diffusion } \\
\text { samplers } \\
\text { were in } \\
\text { wells }\end{array}$ \\
\hline 379 & PW-66 & $\mathrm{C}$ & $779679-0073$ & $779679-0147$ & 29.1 & $11 / 10 / 99$ & $1 / 18 / 00$ & 69 \\
\hline 379 & PW-66 & $\mathrm{D}$ & 779679-0108 & 779679-0148 & 30.8 & $11 / 10 / 99$ & $1 / 18 / 00$ & 69 \\
\hline 379 & PW-66 & $\mathrm{E}$ & 779679-0074 & 779679-0149 & 32.3 & 11/10/99 & $1 / 18 / 00$ & 69 \\
\hline 379 & PW-66 & $\mathrm{F}$ & 779679-0075 & 779679-0150 & 33.9 & $11 / 10 / 99$ & $1 / 18 / 00$ & 69 \\
\hline 379 & PW-66 & F repl & NA & $779679-0152$ & 33.9 & $11 / 10 / 99$ & $1 / 18 / 00$ & 69 \\
\hline Site 11 & MW-12 & A & 779679-0006 & 779679-0012 & 30.5 & $11 / 13 / 99$ & $1 / 17 / 00$ & 65 \\
\hline Site 11 & MW-12 & B & 779679-0007 & 779679-0013 & 32.1 & $11 / 13 / 99$ & $1 / 17 / 00$ & 65 \\
\hline Site 11 & MW-12 & $\mathrm{C}$ & 779679-0008 & 779679-0014 & 33.7 & $11 / 13 / 99$ & $1 / 17 / 00$ & 65 \\
\hline Site 11 & MW-12* & $\mathrm{D}$ & 779679-0009 & 779679-0057 & 35.1 & $11 / 13 / 99$ & $1 / 17 / 00$ & 65 \\
\hline Site 11 & MW-12* & D repl & NA & 779679-0060 & 35.1 & $11 / 13 / 99$ & $1 / 17 / 00$ & 65 \\
\hline Site 11 & MW-12 & $\mathrm{E}$ & 779679-0010 & 779679-0058 & 36.9 & $11 / 13 / 99$ & $1 / 17 / 00$ & 65 \\
\hline Site 11 & MW-12 & $\mathrm{F}$ & 779679-0011 & 779679-0059 & 38.5 & $11 / 13 / 99$ & $1 / 17 / 00$ & 65 \\
\hline Site 11 & MW-5D & A & 779679-0121 & 779679-0128 & 50.8 & $11 / 12 / 99$ & $1 / 18 / 00$ & 67 \\
\hline Site 11 & MW-5D & B & 779679-0122 & 779679-0129 & 52.3 & 11/12/99 & $1 / 18 / 00$ & 67 \\
\hline Site 11 & MW-5D & $\mathrm{C}$ & 779679-0123 & 779679-0130 & 54.2 & $11 / 12 / 99$ & $1 / 18 / 00$ & 67 \\
\hline Site 11 & MW-5D & C repl & NA & 779679-0134 & 54.2 & $11 / 12 / 99$ & $1 / 18 / 00$ & 67 \\
\hline Site 11 & MW-5D* & $\mathrm{D}$ & 779679-0124 & 779679-0131 & 55.75 & $11 / 12 / 99$ & $1 / 18 / 00$ & 67 \\
\hline Site 11 & MW-5D & D repl & 779679-0125 & NA & 55.75 & $11 / 12 / 99$ & $1 / 18 / 00$ & 67 \\
\hline Site 11 & MW-5D & $\mathrm{E}$ & 779679-0126 & 779679-0132 & 57.4 & $11 / 12 / 99$ & $1 / 18 / 00$ & 67 \\
\hline Site 11 & MW-5D & $\mathrm{F}$ & 779679-0127 & 779679-0133 & 59.0 & $11 / 12 / 99$ & $1 / 18 / 00$ & 67 \\
\hline Site 11 & MW9* & NA & 779679-0154 & 779679-0155 & 31 & $11 / 12 / 99$ & $1 / 19 / 00$ & 68 \\
\hline Site 2 & S2-MW6A & A & 779679-0062 & $779679-0135$ & 6.5 & $11 / 13 / 99$ & $1 / 18 / 00$ & 66 \\
\hline Site 2 & S2-MW6A & $\mathrm{B}$ & 779679-0063 & 779679-0136 & 7.85 & 11/13/99 & $1 / 18 / 00$ & 66 \\
\hline Site 2 & S2-MW6A & $\mathrm{C}$ & 779679-0064 & 779679-0137 & 9.2 & $11 / 13 / 99$ & $1 / 18 / 00$ & 66 \\
\hline Site 2 & S2-MW6A & $\mathrm{D}$ & 779679-0065 & 779679-0138 & 10.6 & $11 / 13 / 99$ & $1 / 18 / 00$ & 66 \\
\hline Site 2 & S2-MW6A & $\mathrm{E}$ & 779679-0066 & 779679-0139 & 11.95 & $11 / 13 / 99$ & $1 / 18 / 00$ & 66 \\
\hline Site 2 & S2-MW6A & $\mathrm{F}$ & 779679-0067 & $779679-0140$ & 13.3 & $11 / 13 / 99$ & $1 / 18 / 00$ & 66 \\
\hline Site 2 & S2-MW6A & G & 779679-0068 & 779679-0141 & 14.65 & $11 / 13 / 99$ & $1 / 18 / 00$ & 66 \\
\hline Site 2 & S2-MW6A & $\mathrm{H}$ & 779679-0069 & 779679-0142 & 16.05 & $11 / 13 / 99$ & $1 / 18 / 00$ & 66 \\
\hline Site 2 & S2-MW6A & I & 779679-0070 & $779679-0143$ & 17.5 & $11 / 13 / 99$ & $1 / 18 / 00$ & 66 \\
\hline Site 2 & S2-MW6A & $\mathrm{J}$ & 779679-0071 & 779679-0144 & 18.95 & $11 / 13 / 99$ & $1 / 18 / 00$ & 66 \\
\hline Site 2 & S2-MW6A & $\mathrm{J}$ repl & 779679-0072 & NA & 18.95 & $11 / 13 / 99$ & $1 / 18 / 00$ & 66 \\
\hline
\end{tabular}


Table 3. Comparison of replicate samples collected by diffusion-sampler methodology, Naval Air Station North Island, California, January 2000

[repl, replicate sample; ft bls, feet below land surface; ( $\mu \mathrm{g} / \mathrm{L}$, micrograms per liter; J, estimated value; $\mathrm{U}$, value was below the analytical quantitation limit; 11DCA, 1,1-dichloroethane; 11DCE, 1,1-dichloroethene; $c$ DCE, cis-1,2-dichloroethene; TCE, trichloroethene]

\begin{tabular}{|c|c|c|c|c|c|c|c|c|c|}
\hline $\begin{array}{c}\text { Well } \\
\text { identifier } \\
\text { and } \\
\text { (depth code) }\end{array}$ & $\begin{array}{l}\text { Depth to dif- } \\
\text { fusion sam- } \\
\text { pler } \\
\text { center } \\
\text { (ft bls) }\end{array}$ & $\begin{array}{l}\text { Site or build- } \\
\text { ing designa- } \\
\text { tion }\end{array}$ & $\begin{array}{c}\text { 11DCA } \\
(\mu \mathrm{g} / \mathrm{L})\end{array}$ & $\begin{array}{c}\text { 11DCE } \\
(\mu \mathrm{g} / \mathrm{L})\end{array}$ & $\begin{array}{l}c \mathrm{DCE} \\
(\mu \mathrm{g} / \mathrm{L})\end{array}$ & $\begin{array}{l}\text { Ethyl- } \\
\text { benzene } \\
(\mu \mathrm{g} / \mathrm{L})\end{array}$ & $\begin{array}{c}\text { TCE } \\
(\mu \mathbf{g} / \mathbf{L})\end{array}$ & $\begin{array}{c}\text { Vinyl } \\
\text { chloride }(\mu \mathrm{g} / \\
\text { L) }\end{array}$ & $\begin{array}{l}\text { Total } \\
\text { xylenes } \\
(\mu \mathrm{g} / \mathrm{L})\end{array}$ \\
\hline$\overline{M W-12(D)}$ & 35.1 & Site 11 & $86 \mathrm{~J}$ & 1,500 & 100 & $100 \mathrm{U}$ & 1,800 & $100 \mathrm{U}$ & $100 \mathrm{U}$ \\
\hline MW-12 (D-repl) & 35.1 & Site 11 & $89 \mathrm{~J}$ & 1,500 & 110 & $100 \mathrm{U}$ & 1,700 & $100 \mathrm{U}$ & $100 \mathrm{U}$ \\
\hline MW-5D (C) & 54.2 & Site 11 & 170 & $2,800 \mathrm{E}$ & 61 & $50 \mathrm{U}$ & 930 & $50 \mathrm{U}$ & $50 \mathrm{U}$ \\
\hline MW-5D (C repl) & 54.2 & Site 11 & 170 & $2,900 \mathrm{E}$ & 61 & $50 \mathrm{U}$ & 930 & $50 \mathrm{U}$ & $50 \mathrm{U}$ \\
\hline MW-68C2 (B) & 39.1 & 472 & $2,500 \mathrm{U}$ & 4,100 & $1,000 \mathrm{~J}$ & $2,500 \mathrm{U}$ & 47,000 & $2,500 \mathrm{U}$ & $2,500 \mathrm{U}$ \\
\hline MW-68C2 (B repl) & 39.1 & 472 & $5,000 \mathrm{U}$ & $4,300 \mathrm{~J}$ & $960 \mathrm{~J}$ & $5,000 \mathrm{U}$ & 52,000 & $5,000 \mathrm{U}$ & $5,000 \mathrm{U}$ \\
\hline MW-68C2 (M) & 59.5 & 472 & $500 \mathrm{U}$ & $350 \mathrm{~J}$ & $500 \mathrm{U}$ & $500 \mathrm{U}$ & 7,000 & $500 \mathrm{U}$ & $500 \mathrm{U}$ \\
\hline MW-68C2 (M repl) & 59.5 & 472 & $500 \mathrm{U}$ & $360 \mathrm{~J}$ & $500 \mathrm{U}$ & $500 \mathrm{U}$ & 6,800 & $500 \mathrm{U}$ & $500 \mathrm{U}$ \\
\hline PW-15 (B) & 27.1 & 379 & 52 & 8 & 130 & 15 & 7 & 72 & 52 \\
\hline PW-15 (B repl) & 27.1 & 379 & 54 & 8 & 130 & 17 & $5 \mathrm{~J}$ & 75 & 57 \\
\hline PW-15 (E) & 31.7 & 379 & $500 \mathrm{U}$ & $500 \mathrm{U}$ & 1,900 & $500 \mathrm{U}$ & 5,500 & $500 \mathrm{U}$ & $500 \mathrm{U}$ \\
\hline PW-15 (E repl) & 31.7 & 379 & $500 \mathrm{U}$ & $500 \mathrm{U}$ & 1,900 & $500 \mathrm{U}$ & 5,600 & $500 \mathrm{U}$ & $500 \mathrm{U}$ \\
\hline PW-55 (B) & 28.9 & 379 & $2,500 \mathrm{U}$ & $2,500 \mathrm{U}$ & 6,500 & $2,500 \mathrm{U}$ & 39,000 & $2,500 \mathrm{U}$ & $2,500 \mathrm{U}$ \\
\hline PW-55 (B repl) & 28.9 & 379 & $2,500 \mathrm{U}$ & $2,500 \mathrm{U}$ & 6,700 & $2,500 \mathrm{U}$ & 36,000 & $2,500 \mathrm{U}$ & $2,500 \mathrm{U}$ \\
\hline PW-55 (E) & 33.1 & 379 & $2,500 \mathrm{U}$ & $2,500 \mathrm{U}$ & 6,300 & $2,500 \mathrm{U}$ & 33,000 & $2,500 \mathrm{U}$ & $2,500 \mathrm{U}$ \\
\hline PW-55 (E repl) & 33.1 & 379 & $2,500 \mathrm{U}$ & $2,500 \mathrm{U}$ & 6,100 & $2,500 \mathrm{U}$ & 31,000 & $2,500 \mathrm{U}$ & $2,500 \mathrm{U}$ \\
\hline PW-66 (B) & 28.9 & 379 & $500 \mathrm{U}$ & $500 \mathrm{U}$ & 3,400 & $500 \mathrm{U}$ & 9,000 & $500 \mathrm{U}$ & $500 \mathrm{U}$ \\
\hline PW-66 (B repl) & 28.9 & 379 & $500 \mathrm{U}$ & $500 \mathrm{U}$ & 3,200 & $500 \mathrm{U}$ & 9,200 & $500 \mathrm{U}$ & $500 \mathrm{U}$ \\
\hline PW-66 (F) & 33.1 & 379 & $5 \mathrm{U}$ & 130 & $5 \mathrm{U}$ & $5 \mathrm{U}$ & 13 & $5 \mathrm{U}$ & $5 \mathrm{U}$ \\
\hline PW-66 (F repl) & 33.1 & 379 & $5 \mathrm{U}$ & 120 & $5 \mathrm{U}$ & $5 \mathrm{U}$ & 18 & $5 \mathrm{U}$ & $5 \mathrm{U}$ \\
\hline
\end{tabular}

obtained using the bladder pump (table 7). The reason for the difference in tetrachloroethene concentrations is not known.

The data from well MW-5 (site 11) show that the diffusion samplers performed favorably. At well MW-5, where a bladder pump was used to obtain water adjacent to a diffusion sampler and where peristaltic pumps were used at the other depths, the difference between the TCE concentration in water from the adjacent diffusion sampler and the average concentration $(300 \mu \mathrm{g} / \mathrm{L})$ in water from the bladder pump was relatively small (17-percent difference) (table 7). Moreover, the higher TCE concentration in water from the diffusion sampler compared to the concentration in water from the bladder pump implies that the sample collected by the diffusion method was more discrete than the sample collected by using the bladder pump.
A comparison between diffusion samples and a bladder pump sample at well MW-12 showed that the TCE concentration in water from the diffusion sampler was similar to the TCE concentration in water from the bladder pump $(1,800$ and $2,100 \mu \mathrm{g} / \mathrm{L}$, respectively); however, the cis-1,2-dichloroethene ( $c$ DCE) concentration in water from the diffusion sampler was substantially lower (78 percent) than the concentration in water from the bladder pump (table 7 and fig. 2). This difference may be due to in-well mixing by lowflow sampling in a chemically stratified part of the screened interval. Data from diffusion samplers show that the VOC concentrations substantially increased with depth over a distance of only $3.4 \mathrm{ft}$ and that the bladder pump was positioned at a transition zone between two depths of differing concentrations (table 5 and fig. 2). The bladder pump was sampled 
Table 4. Comparison of replicate samples collected by low-flow methodology, Naval Air Station North Island, California, January 2000

[repl, replicate sample; *, sample collected by using bladder pump - low-flow samples without * were collected by using a peristaltic pump; \#, data from OHM Remediation Services Corp. (2000); ft bls, feet below land surface; $(\mu \mathrm{g} / \mathrm{L}$, micrograms per liter; J, estimated value; $\mathrm{U}$, value was below the analytical quantitation limit; 11DCA, 1,1-dichloroethane; 11DCE, 1,1-dichloroethene; $c$ DCE, cis-1,2 dichloroethene; TCE, trichloroethene]

\begin{tabular}{|c|c|c|c|c|c|c|c|c|c|}
\hline $\begin{array}{l}\text { Well } \\
\text { Identifier and (depth } \\
\text { code) }\end{array}$ & $\begin{array}{c}\text { Depth to } \\
\text { diffusion } \\
\text { sampler } \\
\text { center } \\
\text { (ft bls) }\end{array}$ & $\begin{array}{l}\text { Site or build- } \\
\text { ing } \\
\text { designation }\end{array}$ & $\begin{array}{c}\text { 11DCA } \\
(\mu \mathrm{g} / \mathrm{L})\end{array}$ & $\begin{array}{c}\text { 11DCE } \\
(\mu \mathrm{g} / \mathrm{L})\end{array}$ & $\begin{array}{l}c \mathrm{DCE} \\
(\mu \mathrm{g} / \mathrm{L})\end{array}$ & $\begin{array}{c}\text { Ethyl- } \\
\text { benzene } \\
(\mu \mathrm{g} / \mathrm{L})\end{array}$ & $\begin{array}{c}\text { TCE } \\
(\mu \mathbf{g} / \mathbf{L})\end{array}$ & $\begin{array}{c}\text { Vinyl } \\
\text { chloride } \\
(\mu \mathrm{g} / \mathrm{L})\end{array}$ & $\begin{array}{c}\text { Total } \\
\text { xylenes } \\
(\mu \mathrm{g} / \mathrm{L})\end{array}$ \\
\hline MW-10 (G) & 18.8 & 653 & $5 \mathrm{U}$ & $0 \mathrm{~J}$ & $5 \mathrm{U}$ & $5 \mathrm{U}$ & 6 & $5 \mathrm{U}$ & $3 \mathrm{~J}$ \\
\hline MW-10 (G repl) & 18.8 & 653 & $5 \mathrm{U}$ & $5 \mathrm{U}$ & $1 \mathrm{~J}$ & $5 \mathrm{U}$ & 9 & $5 \mathrm{U}$ & $5 \mathrm{U}$ \\
\hline MW-13B*\# & 26 & 653 & $5 \mathrm{U}$ & $5 \mathrm{U}$ & 3,100 & $5 \mathrm{U}$ & $5 \mathrm{U}$ & 1,600 & $5 \mathrm{U}$ \\
\hline MW-13B*\# (repl) & 26 & 653 & $5 \mathrm{U}$ & $5 \mathrm{U}$ & 3,200 & $5 \mathrm{U}$ & $5 \mathrm{U}$ & 1,400 & $5 \mathrm{U}$ \\
\hline MW-13C (B) & 46.6 & 653 & $5 \mathrm{U}$ & $5 \mathrm{U}$ & $3 \mathrm{~J}$ & $5 \mathrm{U}$ & $5 \mathrm{U}$ & $5 \mathrm{U}$ & $5 \mathrm{U}$ \\
\hline MW-13C (B repl) & 46.6 & 653 & $5 \mathrm{U}$ & $5 \mathrm{U}$ & $2 \mathrm{~J}$ & $5 \mathrm{U}$ & $5 \mathrm{U}$ & $5 \mathrm{U}$ & $5 \mathrm{U}$ \\
\hline MW-5D (D)* & 55.7 & Site 11 & 51 & 760 & $23 \mathrm{~J}$ & $25 \mathrm{U}$ & 320 & $25 \mathrm{U}$ & $25 \mathrm{U}$ \\
\hline MW-5D (D repl)* & 55.7 & Site 11 & 44 & 670 & $22 \mathrm{~J}$ & $25 \mathrm{U}$ & 280 & $25 \mathrm{U}$ & $25 \mathrm{U}$ \\
\hline MW-68C2 (J) & 53.9 & 472 & $2,500 \mathrm{U}$ & $2,500 \mathrm{~J}$ & $2,500 \mathrm{U}$ & $2,500 \mathrm{U}$ & 38,000 & $2,500 \mathrm{U}$ & $2,500 \mathrm{U}$ \\
\hline MW-68C2 (J repl) & 53.9 & 472 & $2,500 \mathrm{U}$ & 2,600 & $2,500 \mathrm{U}$ & $2,500 \mathrm{U}$ & 38,000 & $2,500 \mathrm{U}$ & $2,500 \mathrm{U}$ \\
\hline MW-68B (B) & 37.0 & 472 & $5,000 \mathrm{U}$ & $4,400 \mathrm{~J}$ & $5,000 \mathrm{U}$ & $5,000 \mathrm{U}$ & 34,000 & $5,000 \mathrm{U}$ & $5,000 \mathrm{U}$ \\
\hline MW-68B (B repl) & 37.0 & 472 & $5,000 \mathrm{U}$ & $4,900 \mathrm{~J}$ & $5,000 \mathrm{U}$ & $5,000 \mathrm{U}$ & 33,000 & $5,000 \mathrm{U}$ & $5,000 \mathrm{U}$ \\
\hline PW-55 (E) & 33.1 & 379 & $2,500 \mathrm{U}$ & $2,500 \mathrm{U}$ & 5,500 & $2,500 \mathrm{U}$ & 29,000 & $2,500 \mathrm{U}$ & $2,500 \mathrm{U}$ \\
\hline PW-55 (E repl) & 33.1 & 379 & $2,500 \mathrm{U}$ & $2,500 \mathrm{U}$ & 5,700 & $2,500 \mathrm{U}$ & 29,000 & $2,500 \mathrm{U}$ & $2,500 \mathrm{U}$ \\
\hline S2-MW-6A (J) & 18.9 & Site 2 & $5 \mathrm{U}$ & $5 \mathrm{U}$ & $5 \mathrm{U}$ & $5 \mathrm{U}$ & $5 \mathrm{U}$ & $5 \mathrm{U}$ & $5 \mathrm{U}$ \\
\hline S2-MW-6A (J repl) & 18.9 & Site 2 & $5 \mathrm{U}$ & $5 \mathrm{U}$ & $5 \mathrm{U}$ & $5 \mathrm{U}$ & $5 \mathrm{U}$ & $5 \mathrm{U}$ & $5 \mathrm{U}$ \\
\hline
\end{tabular}

following low-flow sampling from three overlying depths using a peristaltic pump; thus, the concentration interface potentially shifted upward toward the bladder pump intake. It is possible that in-well mixing was more pronounced for $c$ DCE than for TCE because there was a greater percentage of change in concentrations with depth for $c \mathrm{DCE}$ than for TCE. The $c \mathrm{DCE}$ concentration increased by a factor of 26 (100 to $2,600 \mu \mathrm{g} / \mathrm{L}$ ) over a depth of 3.4 feet, whereas TCE increased by only a factor of 4.6 over the same depth interval $(1,700$ to $7,800 \mu \mathrm{g} / \mathrm{L})$ (fig. 2). The VOC concentration data indicate that in well MW-12, the diffusion samplers collected point samples of ground water, whereas the bladder pump either collected water from a greater radius of influence or from water induced up the well bore by low-flow sampling at shallower depths.
Wells MW-13A and MW-13B were tested using diffusion samplers and low-flow sampling with a peristaltic pump. Following sample collection with the peristaltic pump, the diffusion samplers were recovered and the wells were sampled by using a bladder pump (bladder-pump data from OHM Remediation Services Corporation, 2000). The data show that at well MW-13A, the $c$ DCE and vinyl chloride concentrations in water obtained using the bladder pump were within the concentration ranges for water obtained from diffusion samplers that bracketed the depth interval of the bladder pump intake (table 7). At well MW-13B, the $c$ DCE concentration also was within the range measured in those diffusion samplers bracketing the depth of the bladder pump intake (table 7 and fig. 3). Although vinyl chloride concentrations differed between the two methods by 16 to 
$\vec{o} \quad$ Table 5. Concentrations of selected chlorinated volatile organic compounds in water from diffusion and low-flow sampling, Naval Air Station North Island, California, January 2000

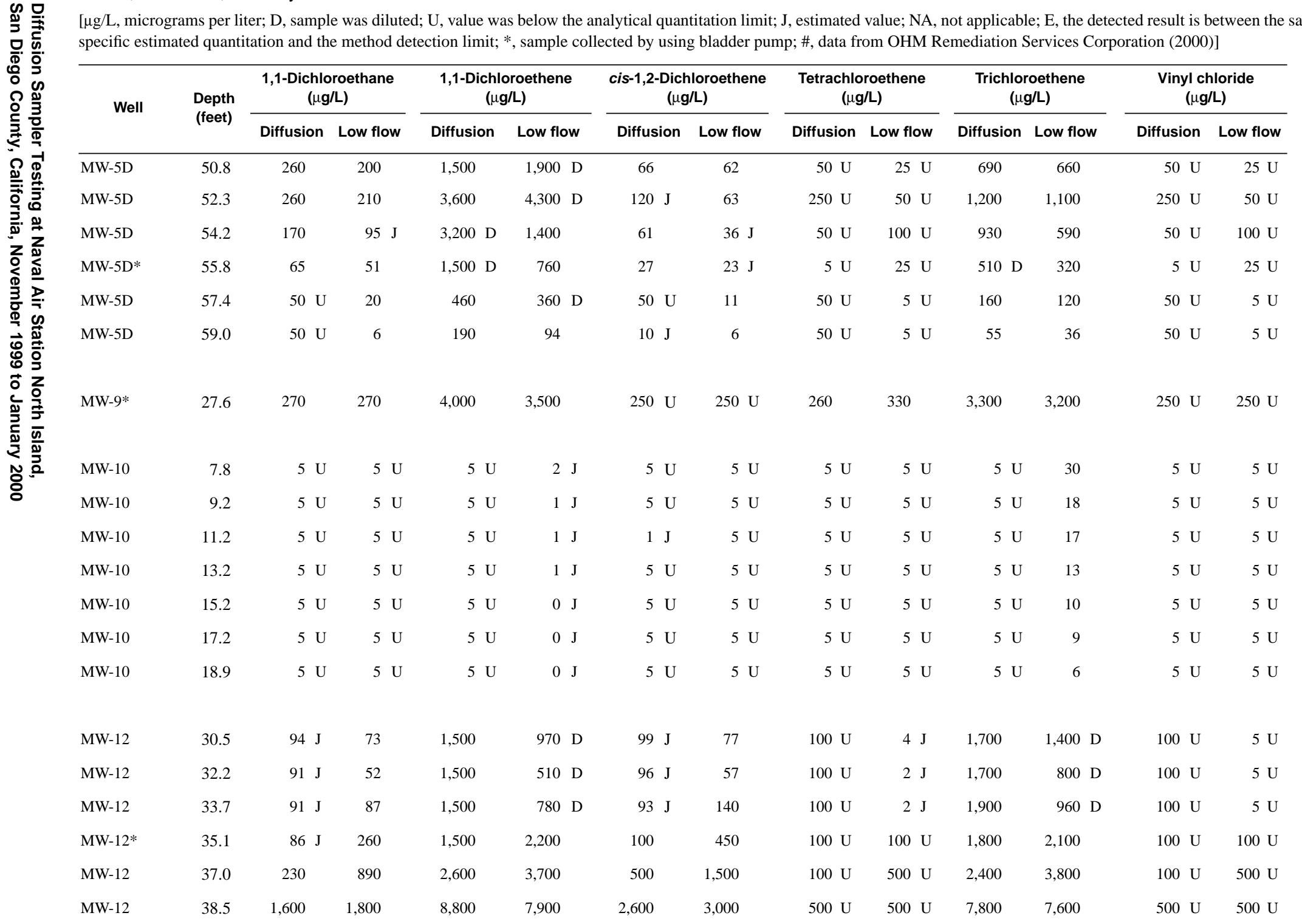


Table 5. Concentrations of selected chlorinated volatile organic compounds in water from diffusion and low-flow sampling, Naval Air Station North Island, California, January 2000-Continued

$[\mu \mathrm{g} / \mathrm{L}$, micrograms per liter; $\mathrm{D}$, sample was diluted; $\mathrm{U}$, value was below the analytical quantitation limit; J, estimated value; NA, not applicable; E, the detected result is between the samplespecific estimated quantitation and the method detection limit; *, sample collected by using bladder pump; \#, data from OHM Remediation Services Corporation (2000)]

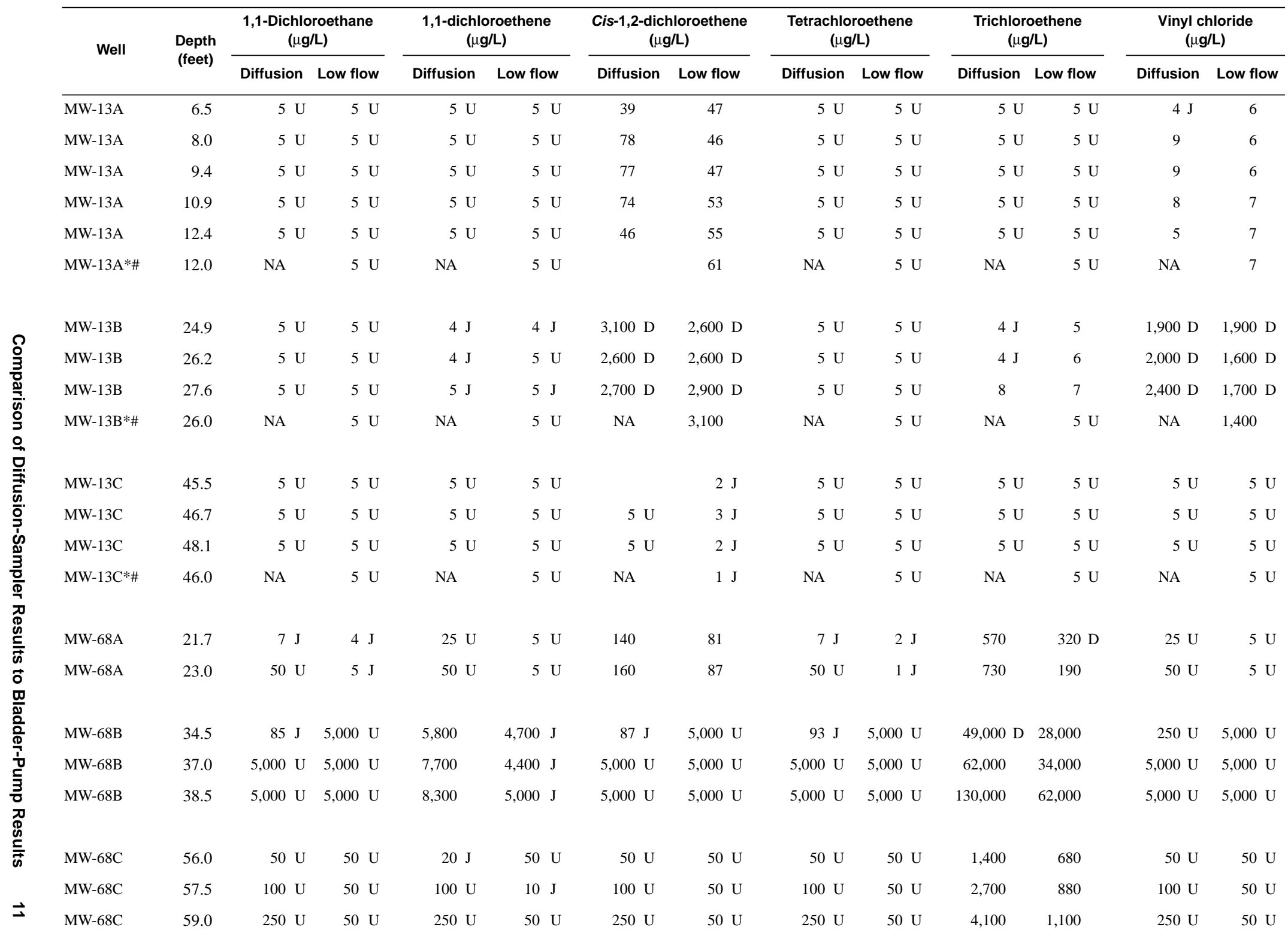


$\vec{N} \quad$ Table 5. Concentrations of selected chlorinated volatile organic compounds in water from diffusion and low-flow sampling, Naval Air Station North Island, California, January 2000-Continued

$[\mu \mathrm{g} / \mathrm{L}$, micrograms per liter; D, sample was diluted; U, value was below the analytical quantitation limit; J, estimated value; NA, not applicable; E, the detected result is between the sample-specific estimated quantitation and the method detection limit; *, sample collected by using bladder pump; \#, data from OHM Remediation Services Corporation (2000)]

\begin{tabular}{|c|c|c|c|c|c|c|c|c|c|c|c|c|c|c|c|}
\hline \multirow{2}{*}{ Well } & \multirow{2}{*}{$\begin{array}{l}\text { Depth } \\
\text { (feet) }\end{array}$} & \multicolumn{2}{|c|}{$\begin{array}{c}\text { 1,1-Dichloroethane } \\
(\mu \mathrm{g} / \mathrm{L})\end{array}$} & \multicolumn{2}{|c|}{$\begin{array}{c}\text { 1,1-Dichloroethene } \\
(\mu \mathrm{g} / \mathrm{L})\end{array}$} & \multicolumn{2}{|c|}{$\begin{array}{c}\text { cis-1,2-Dichloroethene } \\
(\mu \mathrm{g} / \mathrm{L})\end{array}$} & \multicolumn{4}{|c|}{$\begin{array}{c}\text { Tetrachloroethene } \\
(\mu \mathrm{g} / \mathrm{L})\end{array}$} & \multicolumn{2}{|c|}{$\begin{array}{c}\text { Trichloroethene } \\
(\mu \mathrm{g} / \mathrm{L})\end{array}$} & \multicolumn{2}{|c|}{$\begin{array}{l}\text { Vinyl chloride } \\
(\mu \mathrm{g} / \mathrm{L})\end{array}$} \\
\hline & & Diffusion & Low flow & Diffusion & Low flow & Diffusion & Low flow & Diffusion & & Low flov & & Diffusion & Low flow & Diffusion & Low flow \\
\hline MW-68C2 & 37.3 & $1,000 \mathrm{U}$ & $500 \mathrm{U}$ & 2,400 & 1,200 & $490 \mathrm{~J}$ & $360 \mathrm{~J}$ & 1,000 & $\mathrm{U}$ & 500 & $\mathrm{U}$ & 19,000 & 9,100 & $1,000 \mathrm{U}$ & $500 \mathrm{~L}$ \\
\hline MW-68C2 & 39.1 & $2,500 \mathrm{U}$ & $2,500 \mathrm{U}$ & 4,100 & 3,400 & $1,000 \mathrm{~J}$ & $2,500 \mathrm{U}$ & 2,500 & $\mathrm{U}$ & 2,500 & $\mathrm{U}$ & 47,000 & 34,000 & $2,500 \mathrm{U}$ & $2,500 \mathrm{~L}$ \\
\hline MW-68C2 & 40.5 & $5,000 \mathrm{U}$ & $2,500 \mathrm{U}$ & 5,400 & 2,700 & $5,000 \mathrm{U}$ & $2,500 \mathrm{U}$ & 5,000 & $\mathrm{U}$ & 2,500 & $\mathrm{U}$ & 84,000 & 39,000 & $5,000 \mathrm{U}$ & $2,500 \mathrm{U}$ \\
\hline MW-68C2 & 42.1 & $10,000 \mathrm{U}$ & $2,500 \mathrm{U}$ & 14,000 & 2,800 & $10,000 \mathrm{U}$ & $2,500 \mathrm{U}$ & 10,000 & $\mathrm{U}$ & 2,500 & $\mathrm{U}$ & 200,000 & 46,000 & $10,000 \mathrm{U}$ & $2,500 \mathrm{U}$ \\
\hline MW-68C2 & 44.2 & $10,000 \mathrm{U}$ & $2,500 \mathrm{U}$ & $7,800 \mathrm{~J}$ & 3,600 & $10,000 \mathrm{U}$ & $2,500 \mathrm{U}$ & 10,000 & $\mathrm{U}$ & 2,500 & $\mathrm{U}$ & 110,000 & 54,000 & $10,000 \mathrm{U}$ & $2,500 \mathrm{U}$ \\
\hline MW-68C2 & 46.1 & $10,000 \mathrm{U}$ & $1,000 \mathrm{U}$ & $7,500 \mathrm{~J}$ & 1,200 & $10,000 \mathrm{U}$ & $1,000 \mathrm{U}$ & 10,000 & $\mathrm{U}$ & 1,000 & $\mathrm{U}$ & 110,000 & 17,000 & $10,000 \mathrm{U}$ & $1,000 \mathrm{~L}$ \\
\hline MW-68C2 & 47.9 & $10,000 \mathrm{U}$ & $5,000 \mathrm{U}$ & $7,400 \mathrm{~J}$ & $4,300 \mathrm{~J}$ & $10,000 \mathrm{U}$ & $5,000 \mathrm{U}$ & 10,000 & $\mathrm{U}$ & 5,000 & $\mathrm{U}$ & 110,000 & 55,000 & $10,000 \mathrm{U}$ & $5,000 \mathrm{~L}$ \\
\hline MW-68C2 & 49.9 & $10,000 \mathrm{U}$ & $2,500 \mathrm{U}$ & $7,200 \mathrm{~J}$ & 4,400 & $10,000 \mathrm{U}$ & $2,500 \mathrm{U}$ & 10,000 & $\mathrm{U}$ & 2,500 & $\mathrm{U}$ & 100,000 & 53,000 & $10,000 \mathrm{U}$ & $2,500 \mathrm{~L}$ \\
\hline MW-68C2 & 52.0 & $2,500 \mathrm{U}$ & $2,500 \mathrm{U}$ & 3,000 & 2,800 & $2,500 \mathrm{U}$ & $2,500 \mathrm{U}$ & 2,500 & $\mathrm{U}$ & 2,500 & $\mathrm{U}$ & 42,000 & 42,000 & $2,500 \mathrm{U}$ & $2,500 \mathrm{U}$ \\
\hline MW-68C2 & 53.9 & $1,000 \mathrm{U}$ & $2,500 \mathrm{U}$ & 1,100 & $2,500 \mathrm{~J}$ & $1,000 \mathrm{U}$ & $2,500 \mathrm{U}$ & 1,000 & $\mathrm{U}$ & 2,500 & $\mathrm{U}$ & 14,000 & 38,000 & $1,000 \mathrm{U}$ & $2,500 \mathrm{U}$ \\
\hline MW-68C2 & 55.6 & $500 \mathrm{U}$ & $1,000 \mathrm{U}$ & 600 & 1,000 & $500 \mathrm{U}$ & $1,000 \mathrm{U}$ & 500 & $\mathrm{U}$ & 1,000 & $\mathrm{U}$ & 8,800 & 14,000 & $500 \mathrm{U}$ & $1,000 \mathrm{~L}$ \\
\hline MW-68C2 & 57.5 & $500 \mathrm{U}$ & $500 \mathrm{U}$ & $450 \mathrm{~J}$ & 710 & $500 \mathrm{U}$ & $500 \mathrm{U}$ & 500 & $\mathrm{U}$ & 500 & $\mathrm{U}$ & 7,300 & 9,100 & $500 \mathrm{U}$ & $500 \mathrm{~L}$ \\
\hline MW-68C2 & 59.5 & $500 \mathrm{U}$ & $500 \mathrm{U}$ & $350 \mathrm{~J}$ & 920 & $500 \mathrm{U}$ & $500 \mathrm{U}$ & 500 & $\mathrm{U}$ & 500 & $\mathrm{U}$ & 7,000 & 11,000 & $500 \mathrm{U}$ & $500 \mathrm{~L}$ \\
\hline MW-68C2 & 61.5 & $500 \mathrm{U}$ & $500 \mathrm{U}$ & $280 \mathrm{~J}$ & 740 & $500 \mathrm{U}$ & $500 \mathrm{U}$ & 500 & $\mathrm{U}$ & 500 & $\mathrm{U}$ & 6,500 & 11,000 & $500 \mathrm{U}$ & $500 \mathrm{U}$ \\
\hline PW-15 & 25.4 & 45 & 53 & $4 \mathrm{~J}$ & 12 & 42 & $200 \mathrm{E}$ & 5 & $\mathrm{U}$ & 2. & $\mathrm{~J}$ & $4 \mathrm{~J}$ & 15 & 82 & 66 \\
\hline PW-15 & 27.1 & 52 & 52 & $\bar{D}$ & 22 & 130 & $850 \mathrm{D}$ & $1 \mathrm{~J}$ & $\mathrm{~J}$ & 8 & & 7 & 92 & 72 & 29 \\
\hline PW-15 & 28.5 & $73 \mathrm{~J}$ & $77 \mathrm{~J}$ & $36 \mathrm{~J}$ & $66 \mathrm{~J}$ & 1,500 & 2,200 & $100 \mathrm{~J}$ & & 250 & $\mathrm{U}$ & 180 & 2,500 & $100 \mathrm{U}$ & $250 \mathrm{U}$ \\
\hline PW-15 & 30.2 & $97 \mathrm{~J}$ & $500 \mathrm{U}$ & $250 \mathrm{U}$ & $500 \mathrm{U}$ & 2,900 & 1,600 & $59 \mathrm{~J}$ & $\mathrm{~J}$ & 500 & $\mathrm{U}$ & 3,000 & 4,700 & $250 \mathrm{U}$ & $500 \mathrm{U}$ \\
\hline PW-15 & 31.8 & $500 \mathrm{U}$ & $500 \mathrm{U}$ & $500 \mathrm{U}$ & $500 \mathrm{U}$ & 1,900 & 1,800 & 500 & $\mathrm{U}$ & 500 & $\mathrm{U}$ & 5,500 & 5,800 & $500 \mathrm{U}$ & $500 \mathrm{U}$ \\
\hline PW-15 & 33.2 & $500 \mathrm{U}$ & $500 \mathrm{U}$ & $500 \mathrm{U}$ & $500 \mathrm{U}$ & 2,400 & 2,100 & $120 \mathrm{~J}$ & $\mathrm{~J}$ & 130 & $\mathrm{~J}$ & 7,500 & 7,300 & $500 \mathrm{U}$ & $500 \mathrm{U}$ \\
\hline PW-55 & 27.1 & $2,500 \mathrm{U}$ & $2,500 \mathrm{U}$ & $2,500 \mathrm{U}$ & $2,500 \mathrm{U}$ & 7,300 & 5,700 & 2,500 & $\mathrm{U}$ & 2,500 & $\mathrm{U}$ & 39,000 & 31,000 & $2,500 \mathrm{U}$ & $2,500 \mathrm{U}$ \\
\hline PW-55 & 28.9 & $2,500 \mathrm{U}$ & $2,500 \mathrm{U}$ & $2,500 \mathrm{U}$ & $2,500 \mathrm{U}$ & 6,500 & 5,900 & 2,500 & $\mathrm{U}$ & 2,500 & $\mathrm{U}$ & 39,000 & 32,000 & $2,500 \mathrm{U}$ & $2,500 \mathrm{U}$ \\
\hline PW-55 & 30.6 & $2,500 \mathrm{U}$ & $2,500 \mathrm{U}$ & $2,500 \mathrm{U}$ & $2,500 \mathrm{U}$ & 6,600 & 5,900 & 2,500 & $\mathrm{U}$ & 2,500 & $\mathrm{U}$ & 38,000 & 34,000 & $2,500 \mathrm{U}$ & $2,500 \mathrm{U}$ \\
\hline
\end{tabular}


Table 5. Concentrations of selected chlorinated volatile organic compounds in water from diffusion and low-flow sampling, Naval Air Station North Island, California, January 2000-Continued

$[\mu \mathrm{g} / \mathrm{L}$, micrograms per liter; D, sample was diluted; U, value was below the analytical quantitation limit; J, estimated value; NA, not applicable; E, the detected result is between the sample-specific estimated quantitation and the method detection limit; *, sample collected by using bladder pump; \#, data from OHM Remediation Services Corporation (2000)]

\begin{tabular}{|c|c|c|c|c|c|c|c|c|c|c|c|c|c|}
\hline \multirow{2}{*}{ Well } & \multirow{2}{*}{$\begin{array}{l}\text { Depth } \\
\text { (feet) }\end{array}$} & \multicolumn{2}{|c|}{$\begin{array}{c}\text { 1,1-Dichloroethane } \\
(\mu \mathrm{g} / \mathrm{L})\end{array}$} & \multicolumn{2}{|c|}{$\begin{array}{c}\text { 1,1-Dichloroethene } \\
(\mu \mathrm{g} / \mathrm{L})\end{array}$} & \multicolumn{2}{|c|}{$\begin{array}{c}\text { cis-1,2-Dichloroethene } \\
(\mu \mathrm{g} / \mathrm{L})\end{array}$} & \multicolumn{2}{|c|}{$\begin{array}{c}\text { Tetrachloroethene } \\
(\mu \mathrm{g} / \mathrm{L})\end{array}$} & \multicolumn{2}{|c|}{$\begin{array}{l}\text { Trichloroethene } \\
(\mu \mathrm{g} / \mathrm{L})\end{array}$} & \multicolumn{2}{|c|}{$\begin{array}{l}\text { Vinyl chloride } \\
(\mu \mathrm{g} / \mathrm{L})\end{array}$} \\
\hline & & Diffusion & Low flow & Diffusion & Low flow & Diffusion & Low flow & Diffusion & Low flow & Diffusion & Low flow & Diffusio & Low flo \\
\hline PW-55 & 31.9 & $2,500 \mathrm{U}$ & $2,500 \mathrm{U}$ & $2,500 \mathrm{U}$ & $2,500 \mathrm{U}$ & 6,800 & 5,300 & $2,500 \mathrm{U}$ & $2,500 \mathrm{U}$ & 38,000 & 29,000 & 2,500 & 2,500 \\
\hline PW-55 & 33.1 & $2,500 \mathrm{U}$ & $2,500 \mathrm{U}$ & $2,500 \mathrm{U}$ & $2,500 \mathrm{U}$ & 6,300 & 5,500 & $2,500 \mathrm{U}$ & $2,500 \mathrm{U}$ & 33,000 & 29,000 & 2,500 & 2,500 \\
\hline PW-66 & 25.5 & $1,000 \mathrm{U}$ & $500 \mathrm{U}$ & $1,000 \mathrm{U}$ & $38 \mathrm{~J}$ & 2,000 & 1,600 & $1,000 \mathrm{U}$ & $599 \mathrm{U}$ & 17,000 & 13,000 & 1,000 & 500 \\
\hline PW-66 & 27.3 & $500 \mathrm{U}$ & $500 \mathrm{U}$ & $500 \mathrm{U}$ & $500 \mathrm{U}$ & 3,400 & 1,000 & $500 \mathrm{U}$ & $500 \mathrm{U}$ & 9,000 & 6,400 & 500 & 500 \\
\hline PW-66 & 29.1 & $50 \mathrm{U}$ & $500 \mathrm{U}$ & $38 \mathrm{~J}$ & $500 \mathrm{U}$ & $23 \mathrm{~J}$ & $500 \mathrm{U}$ & $50 \mathrm{U}$ & $500 \mathrm{U}$ & 770 & 9,800 & 50 & 500 \\
\hline PW-66 & 30.8 & $25 \mathrm{U}$ & $500 \mathrm{U}$ & 49 & $500 \mathrm{U}$ & $25 \mathrm{U}$ & $500 \mathrm{U}$ & $25 \mathrm{U}$ & $500 \mathrm{U}$ & 180 & 5,600 & 25 & 500 \\
\hline PW-66 & 32.3 & $5 \mathrm{U}$ & $500 \mathrm{U}$ & 72 & $500 \mathrm{U}$ & $5 \mathrm{U}$ & $500 \mathrm{U}$ & $5 \mathrm{U}$ & $500 \mathrm{U}$ & 48 & 6,200 & 5 & 500 \\
\hline PW-66 & 33.9 & $5 \mathrm{U}$ & $500 \mathrm{U}$ & 130 & $500 \mathrm{U}$ & $5 \mathrm{U}$ & $500 \mathrm{U}$ & $1 \mathrm{~J}$ & $500 \mathrm{U}$ & 13 & 6,000 & 5 & 500 \\
\hline S2-MW-6A & 6.5 & $5 \mathrm{U}$ & $5 \mathrm{U}$ & $5 \mathrm{U}$ & $5 \mathrm{U}$ & $5 \mathrm{U}$ & $5 \mathrm{U}$ & $5 \mathrm{U}$ & $5 \mathrm{U}$ & 5 & $5 \mathrm{~L}$ & 5 & 5 \\
\hline S2-MW-6A & 7.9 & $5 \mathrm{U}$ & $5 \mathrm{U}$ & $5 \mathrm{U}$ & $5 \mathrm{U}$ & $5 \mathrm{U}$ & $5 \mathrm{U}$ & $5 \mathrm{U}$ & $5 \mathrm{U}$ & 5 & $2 \mathrm{~L}$ & 5 & 5 \\
\hline S2-MW-6A & 9.2 & $5 \mathrm{U}$ & $5 \mathrm{U}$ & $5 \mathrm{U}$ & $5 \mathrm{U}$ & $5 \mathrm{U}$ & 5 & & & & & & \\
\hline S2-MW-6A & 10.6 & $5 \mathrm{U}$ & $5 \mathrm{U}$ & $5 \mathrm{U}$ & $5 \mathrm{U}$ & $5 \mathrm{U}$ & $5 \mathrm{U}$ & $5 \mathrm{U}$ & $5 \mathrm{U}$ & 2 & $2 \mathrm{~J}$ & 5 & 5 \\
\hline S2-MW-6A & 12.0 & $5 \mathrm{U}$ & $5 \mathrm{U}$ & $5 \mathrm{U}$ & $5 \mathrm{U}$ & $5 \mathrm{U}$ & $5 \mathrm{U}$ & $5 \mathrm{U}$ & $5 \mathrm{U}$ & 5 & $2 \mathrm{~J}$ & 5 & 5 \\
\hline S2-MW-6A & 13.3 & $5 \mathrm{U}$ & $5 \mathrm{U}$ & $5 \mathrm{U}$ & $5 \mathrm{U}$ & $5 \mathrm{U}$ & $5 \mathrm{U}$ & $5 \mathrm{U}$ & $5 \mathrm{U}$ & 1. & $5 \mathrm{~L}$ & 5 & 5 \\
\hline S2-MW-6A & 14.7 & $5 \mathrm{U}$ & $5 \mathrm{U}$ & $5 \mathrm{U}$ & $5 \mathrm{U}$ & $5 \mathrm{U}$ & $5 \mathrm{U}$ & $5 \mathrm{U}$ & $5 \mathrm{U}$ & 5 & $2 \mathrm{~J}$ & 5 & 5 \\
\hline S2-MW-6A & 16.1 & $5 \mathrm{U}$ & $5 \mathrm{U}$ & $5 \mathrm{U}$ & $5 \mathrm{U}$ & $5 \mathrm{U}$ & $5 \mathrm{U}$ & $5 \mathrm{U}$ & $5 \mathrm{U}$ & 5 & $2 \mathrm{~J}$ & 5 & 5 \\
\hline S2-MW-6A & 17.5 & $5 \mathrm{U}$ & $5 \mathrm{U}$ & $5 \mathrm{U}$ & $5 \mathrm{U}$ & $5 \mathrm{U}$ & $5 \mathrm{U}$ & $5 \mathrm{U}$ & $5 \mathrm{U}$ & 5 & $2 \mathrm{~J}$ & 5 & 5 \\
\hline S2-MW-6A & 19.0 & $5 \mathrm{U}$ & $5 \mathrm{U}$ & $5 \mathrm{U}$ & $5 \mathrm{U}$ & $5 \mathrm{U}$ & $5 \mathrm{U}$ & $5 \mathrm{U}$ & $5 \mathrm{U}$ & 5 & $5 \mathrm{~L}$ & 5 & 5 \\
\hline
\end{tabular}


Table 6. Concentrations of benzene, ethylbenzene, toluene, and total xylenes in water from diffusion and low-flow sampling, Naval Air Station North Island, California, January 2000

[ $\mu \mathrm{g} / \mathrm{L}$, micrograms per liter; U, value was below the analytical quantitation limit; J, estimated value; NA, not applicable; *, sample collected by using bladder pump; \#, data from OHM Remediation Services Corporation (2000)]

\begin{tabular}{|c|c|c|c|c|c|c|c|c|c|c|c|c|c|c|}
\hline \multirow[t]{2}{*}{ Well } & \multirow{2}{*}{$\begin{array}{l}\text { Depth } \\
\text { (feet) }\end{array}$} & \multicolumn{3}{|c|}{$\begin{array}{c}\text { Benzene } \\
(\mu \mathrm{g} / \mathrm{L})\end{array}$} & \multicolumn{3}{|c|}{$\begin{array}{l}\text { Ethylbenzene } \\
(\mu \mathrm{g} / \mathrm{L})\end{array}$} & \multicolumn{3}{|c|}{$\begin{array}{l}\text { Toluene } \\
(\mu \mathrm{g} / \mathrm{L})\end{array}$} & \multicolumn{4}{|c|}{$\begin{array}{c}\text { Total xylenes } \\
(\mu \mathrm{g} / \mathrm{L})\end{array}$} \\
\hline & & Diffusion & Low fl & low & Diffus & ion & Low flow & Diffus & sion & Low flow & Diffus & sion & Low & flor \\
\hline MW-5D & 50.8 & $50 \mathrm{U}$ & 25 & $\mathrm{U}$ & 50 & $\mathrm{U}$ & $25 \mathrm{U}$ & 50 & $\mathrm{U}$ & $25 \mathrm{U}$ & & $\mathrm{U}$ & 25 & $\mathrm{U}$ \\
\hline MW-5D & 52.3 & $250 \mathrm{U}$ & 50 & $\mathrm{U}$ & 250 & $\mathrm{U}$ & $50 \mathrm{U}$ & 250 & $\mathrm{U}$ & $50 \mathrm{U}$ & 250 & $\mathrm{U}$ & 50 & $\mathrm{U}$ \\
\hline MW-5D & 54.2 & $50 \mathrm{U}$ & 100 & $\mathrm{U}$ & 50 & $\mathrm{U}$ & $100 \mathrm{U}$ & 50 & $\mathrm{U}$ & $100 \mathrm{U}$ & 50 & $\mathrm{U}$ & 100 & $\mathrm{U}$ \\
\hline MW-5D* & 55.8 & $5 \mathrm{U}$ & 25 & $\mathrm{U}$ & 5 & $\mathrm{U}$ & $25 \mathrm{U}$ & 5 & $\mathrm{U}$ & $25 \mathrm{U}$ & 5 & $\mathrm{U}$ & 25 & $\mathrm{U}$ \\
\hline MW-5D & 57.4 & $50 \mathrm{U}$ & & $\mathrm{U}$ & 50 & $\mathrm{U}$ & $5 \mathrm{U}$ & 50 & $\mathrm{U}$ & $5 \mathrm{U}$ & 50 & $\mathrm{U}$ & 5 & $\mathrm{U}$ \\
\hline MW-5D & 59.0 & $50 \mathrm{U}$ & & $\mathrm{U}$ & 50 & $\mathrm{U}$ & $5 \mathrm{U}$ & 50 & $\mathrm{U}$ & $5 \mathrm{U}$ & 50 & $\mathrm{U}$ & 5 & $\mathrm{U}$ \\
\hline MW-9* & 27.6 & $250 \mathrm{U}$ & 250 & $\mathrm{U}$ & 250 & $\mathrm{U}$ & $250 \mathrm{U}$ & 250 & $\mathrm{U}$ & $250 \mathrm{U}$ & 250 & $\mathrm{U}$ & 250 & $\mathrm{U}$ \\
\hline MW-10 & 7.8 & $5 \mathrm{U}$ & & $\mathrm{U}$ & 5 & $\mathrm{U}$ & $5 \mathrm{U}$ & 5 & $\mathrm{U}$ & $5 \mathrm{U}$ & 5 & $\mathrm{U}$ & 5 & $\mathrm{U}$ \\
\hline MW-10 & 9.2 & $5 \mathrm{U}$ & 5 & $\mathrm{U}$ & 5 & $\mathrm{U}$ & $5 \mathrm{U}$ & 5 & $\mathrm{U}$ & $5 \mathrm{U}$ & 5 & $\mathrm{U}$ & 5 & $\mathrm{U}$ \\
\hline MW-10 & 11.2 & $5 \mathrm{U}$ & 5 & $\mathrm{U}$ & 5 & $\mathrm{U}$ & $5 \mathrm{U}$ & 5 & $\mathrm{U}$ & $5 \mathrm{U}$ & 5 & $\mathrm{U}$ & 5 & $\mathrm{U}$ \\
\hline MW-10 & 13.2 & $5 \mathrm{U}$ & 5 & $\mathrm{U}$ & 5 & $\mathrm{U}$ & $5 \mathrm{U}$ & 5 & $\mathrm{U}$ & $5 \mathrm{U}$ & & $\mathrm{J}$ & 5 & $\mathrm{U}$ \\
\hline MW-10 & 15.2 & $5 \mathrm{U}$ & 5 & $\mathrm{U}$ & 5 & $\mathrm{U}$ & $5 \mathrm{U}$ & 5 & $\mathrm{U}$ & $5 \mathrm{U}$ & 5 & $\mathrm{U}$ & 5 & $\mathrm{U}$ \\
\hline MW-10 & 17.2 & $5 \mathrm{U}$ & 5 & $\mathrm{U}$ & 5 & $\mathrm{U}$ & $5 \mathrm{U}$ & 5 & $\mathrm{U}$ & $5 \mathrm{U}$ & 5 & $\mathrm{U}$ & 5 & $\mathrm{U}$ \\
\hline MW-10 & 18.9 & $5 \mathrm{U}$ & 5 & $\mathrm{U}$ & 5 & $\mathrm{U}$ & $5 \mathrm{U}$ & 5 & $\mathrm{U}$ & $5 \mathrm{U}$ & 5 & $\mathrm{U}$ & & $5 \mathrm{~J}$ \\
\hline MW-12 & 30.5 & $100 \mathrm{U}$ & 5 & $\mathrm{U}$ & 100 & $\mathrm{U}$ & $5 \mathrm{U}$ & 100 & $\mathrm{U}$ & $5 \mathrm{U}$ & 100 & $\mathrm{U}$ & 5 & $\mathrm{U}$ \\
\hline MW-12 & 32.2 & $100 \mathrm{U}$ & 5 & $\mathrm{U}$ & 100 & $\mathrm{U}$ & $5 \mathrm{U}$ & 100 & $\mathrm{U}$ & $5 \mathrm{U}$ & 100 & $\mathrm{U}$ & 5 & $\mathrm{U}$ \\
\hline MW-12 & 33.7 & $100 \mathrm{U}$ & 5 & $\mathrm{U}$ & 100 & $\mathrm{U}$ & $5 \mathrm{U}$ & 100 & $\mathrm{U}$ & $5 \mathrm{U}$ & 100 & $\mathrm{U}$ & 5 & $\mathrm{U}$ \\
\hline MW-12* & 35.1 & $100 \mathrm{U}$ & 100 & $\mathrm{U}$ & 100 & $\mathrm{U}$ & $100 \mathrm{U}$ & 100 & $\mathrm{U}$ & $100 \mathrm{U}$ & 100 & $\mathrm{U}$ & 100 & $U$ \\
\hline MW-12 & 37.0 & $100 \mathrm{U}$ & 500 & $\mathrm{U}$ & 100 & $\mathrm{U}$ & $120 \mathrm{U}$ & 100 & $\mathrm{U}$ & $500 \mathrm{U}$ & 100 & $\mathrm{U}$ & 360 & $\mathrm{U}$ \\
\hline MW-12 & 38.5 & $500 \mathrm{U}$ & 500 & $\mathrm{U}$ & 500 & $\mathrm{U}$ & $500 \mathrm{U}$ & 500 & $\mathrm{U}$ & $500 \mathrm{U}$ & 500 & $\mathrm{U}$ & 500 & $\mathrm{U}$ \\
\hline MW-13A & 6.5 & $5 \mathrm{U}$ & 5 & $\mathrm{U}$ & 5 & $\mathrm{U}$ & $5 \mathrm{U}$ & 5 & $\mathrm{U}$ & $5 \mathrm{U}$ & 5 & $\mathrm{U}$ & 5 & $\mathrm{U}$ \\
\hline MW-13A & 8.0 & $5 \mathrm{U}$ & 5 & $\mathrm{U}$ & 5 & $\mathrm{U}$ & $5 \mathrm{U}$ & 5 & $\mathrm{U}$ & $5 \mathrm{U}$ & 5 & $\mathrm{U}$ & 5 & $\mathrm{U}$ \\
\hline MW-13A & 9.4 & $5 \mathrm{U}$ & 5 & $\mathrm{U}$ & 5 & $\mathrm{U}$ & $5 \mathrm{U}$ & 5 & $\mathrm{U}$ & $5 \mathrm{U}$ & 5 & $\mathrm{U}$ & 5 & $\mathrm{U}$ \\
\hline MW-13A & 10.9 & $5 \mathrm{U}$ & 5 & $\mathrm{U}$ & 5 & $\mathrm{U}$ & $5 \mathrm{U}$ & 5 & $\mathrm{U}$ & $5 \mathrm{U}$ & 5 & $\mathrm{U}$ & 5 & $\mathrm{U}$ \\
\hline MW-13A & 12.4 & $5 \mathrm{U}$ & 5 & $\mathrm{U}$ & 5 & $\mathrm{U}$ & $5 \mathrm{U}$ & 5 & $\mathrm{U}$ & $5 \mathrm{U}$ & 5 & $\mathrm{U}$ & 5 & $\mathrm{U}$ \\
\hline MW-13A*\# & 12.0 & NA & 5 & $\mathrm{U}$ & NA & & $5 \mathrm{U}$ & NA & & $5 \mathrm{U}$ & NA & & & \\
\hline MW-13B & 24.9 & 9 & 5 & & 5 & $\mathrm{U}$ & $5 \mathrm{U}$ & 5 & $\mathrm{U}$ & $5 \mathrm{U}$ & 5 & $\mathrm{U}$ & 5 & $\mathrm{U}$ \\
\hline MW-13B & 26.2 & 5 & & $\mathrm{~J}$ & 5 & $\mathrm{U}$ & $5 \mathrm{U}$ & 5 & $\mathrm{U}$ & $5 \mathrm{U}$ & 5 & $\mathrm{U}$ & 5 & $\mathrm{U}$ \\
\hline MW-13B & 27.6 & $1 \mathrm{~J}$ & & $\mathrm{~J}$ & 5 & $\mathrm{U}$ & $5 \mathrm{U}$ & 1 & $\mathrm{~J}$ & $5 \mathrm{U}$ & 5 & $\mathrm{U}$ & 5 & $\mathrm{U}$ \\
\hline MW13B*\# & 26.0 & NA & 5 & $\mathrm{U}$ & NA & & $5 \mathrm{U}$ & NA & & $5 \mathrm{U}$ & NA & & 5 & $\mathrm{U}$ \\
\hline MW-13C & 45.5 & $5 \mathrm{U}$ & 5 & $\mathrm{U}$ & 5 & $\mathrm{U}$ & $5 \mathrm{U}$ & 5 & $\mathrm{U}$ & $5 \mathrm{U}$ & 5 & $\mathrm{U}$ & 5 & $\mathrm{U}$ \\
\hline MW-13C & 46.7 & $5 \mathrm{U}$ & 5 & $\mathrm{U}$ & 5 & $\mathrm{U}$ & $5 \mathrm{U}$ & 5 & $\mathrm{U}$ & $5 \mathrm{U}$ & 5 & $\mathrm{U}$ & 5 & $\mathrm{U}$ \\
\hline
\end{tabular}


Table 6. Concentrations of benzene, ethylbenzene, toluene, and total xylenes in water from diffusion and low-flow sampling, Naval Air Station North Island, California, January 2000-Continued

[ $\mu \mathrm{g} / \mathrm{L}$, micrograms per liter; U, value was below the analytical quantitation limit; J, estimated value; NA, not applicable; *, sample collected by using bladder pump; \#, data from OHM Remediation Services Corporation (2000)]

\begin{tabular}{|c|c|c|c|c|c|c|c|c|c|c|c|c|c|c|}
\hline \multirow[t]{2}{*}{ Well } & \multirow{2}{*}{$\begin{array}{l}\text { Depth } \\
\text { (feet) }\end{array}$} & \multicolumn{3}{|c|}{$\begin{array}{c}\text { Benzene } \\
(\mu \mathrm{g} / \mathrm{L})\end{array}$} & \multicolumn{3}{|c|}{$\begin{array}{l}\text { Ethylbenzene } \\
(\mu \mathrm{g} / \mathrm{L})\end{array}$} & \multicolumn{4}{|c|}{$\begin{array}{c}\text { Toluene } \\
(\mu \mathrm{g} / \mathrm{L})\end{array}$} & \multicolumn{3}{|c|}{$\begin{array}{l}\text { Total xylenes } \\
(\mu \mathrm{g} / \mathrm{L})\end{array}$} \\
\hline & & Diffusion & Low $\mathrm{fl}$ & low & Diffusion & Low $f$ & flow & Diffus & sion & Low $f$ & low & Diffusion & Low & flow \\
\hline MW-13C & 48.1 & $5 \mathrm{U}$ & & $\mathrm{U}$ & $5 \mathrm{U}$ & & $\mathrm{U}$ & & $\mathrm{U}$ & & $\mathrm{U}$ & $5 \mathrm{U}$ & & $\mathrm{U}$ \\
\hline MW-13C*\# & 46.0 & NA & & $\mathrm{U}$ & NA & & $\mathrm{U}$ & NA & & & $\mathrm{U}$ & NA & & $\mathrm{U}$ \\
\hline MW-68A & 21.7 & $25 \mathrm{U}$ & 5 & $\mathrm{U}$ & $25 \mathrm{U}$ & 5 & $\mathrm{U}$ & 25 & $\mathrm{U}$ & & $\mathrm{U}$ & $25 \mathrm{U}$ & & $\mathrm{U}$ \\
\hline MW-68A & 23.0 & $50 \mathrm{U}$ & 5 & $\mathrm{U}$ & $50 \mathrm{U}$ & 5 & $\mathrm{U}$ & 50 & $\mathrm{U}$ & & $\mathrm{U}$ & $50 \mathrm{U}$ & 5 & $\mathrm{U}$ \\
\hline MW-68B & 34.5 & $250 \mathrm{U}$ & 5,000 & $\mathrm{U}$ & $250 \mathrm{U}$ & 5,000 & $\mathrm{U}$ & 250 & $\mathrm{U}$ & 5,000 & $\mathrm{U}$ & $250 \mathrm{U}$ & 5,000 & $\mathrm{U}$ \\
\hline MW-68B & 37.0 & $5,000 \mathrm{U}$ & 5,000 & $\mathrm{U}$ & $5,000 \mathrm{U}$ & 5,000 & $\mathrm{U}$ & 5,000 & $\mathrm{U}$ & 5,000 & $\mathrm{U}$ & $5,000 \mathrm{U}$ & 5,000 & $\mathrm{U}$ \\
\hline MW-68B & 38.5 & $5,000 \mathrm{U}$ & 5,000 & $\mathrm{U}$ & $5,000 \mathrm{U}$ & 5,000 & $\mathrm{U}$ & 5,000 & $\mathrm{U}$ & 5,000 & $\mathrm{U}$ & $5,000 \mathrm{U}$ & 5,000 & $\mathrm{U}$ \\
\hline MW-68C & 56.0 & $50 \mathrm{U}$ & 50 & $\mathrm{U}$ & $50 \mathrm{U}$ & 50 & $\mathrm{U}$ & 50 & $\mathrm{U}$ & 50 & $\mathrm{U}$ & $50 \mathrm{U}$ & 50 & $\mathrm{U}$ \\
\hline MW-68C & 57.5 & $100 \mathrm{U}$ & 50 & $\mathrm{U}$ & $100 \mathrm{U}$ & 50 & $\mathrm{U}$ & 100 & $\mathrm{U}$ & 50 & $\mathrm{U}$ & $100 \mathrm{U}$ & 50 & $\mathrm{U}$ \\
\hline MW-68C & 59.0 & $250 \mathrm{U}$ & 12 & $\mathrm{~J}$ & $250 \mathrm{U}$ & 50 & $\mathrm{U}$ & 250 & $\mathrm{U}$ & 50 & $\mathrm{U}$ & $250 \mathrm{U}$ & 50 & $\mathrm{U}$ \\
\hline MW-68C2 & 37.3 & $1,000 \mathrm{U}$ & 500 & $\mathrm{U}$ & $1,000 \mathrm{U}$ & 500 & $\mathrm{U}$ & 1,000 & $\mathrm{U}$ & 500 & $\mathrm{U}$ & $1,000 \mathrm{U}$ & 500 & $\mathrm{U}$ \\
\hline MW-68C2 & 39.1 & $2,500 \mathrm{U}$ & 2,500 & $\mathrm{U}$ & $2,500 \mathrm{U}$ & 2,500 & $\mathrm{U}$ & 2,500 & $\mathrm{U}$ & 2,500 & $\mathrm{U}$ & $2,500 \mathrm{U}$ & 2,500 & $\mathrm{U}$ \\
\hline MW-68C2 & 40.5 & $5,000 \mathrm{U}$ & 2,500 & $\mathrm{U}$ & $5,000 \mathrm{U}$ & 2,500 & $\mathrm{U}$ & 5,000 & $\mathrm{U}$ & 2,500 & $\mathrm{U}$ & $5,000 \mathrm{U}$ & 2,500 & $\mathrm{U}$ \\
\hline MW-68C2 & 42.1 & $10,000 \mathrm{U}$ & 2,500 & $\mathrm{U}$ & $10,000 \mathrm{U}$ & 2,500 & $\mathrm{U}$ & 10,000 & $\mathrm{U}$ & 2,500 & $\mathrm{U}$ & $10,000 \mathrm{U}$ & 2,500 & $\mathrm{U}$ \\
\hline MW-68C2 & 44.2 & $10,000 \mathrm{U}$ & 2,500 & $\mathrm{U}$ & $10,000 \mathrm{U}$ & 2,500 & $\mathrm{U}$ & 10,000 & $\mathrm{U}$ & 2,500 & $\mathrm{U}$ & $10,000 \mathrm{U}$ & 2,500 & $\mathrm{U}$ \\
\hline MW-68C2 & 46.1 & $10,000 \mathrm{U}$ & 1,000 & $\mathrm{U}$ & $10,000 \mathrm{U}$ & 1,000 & $\mathrm{U}$ & 10,000 & $\mathrm{U}$ & 1,000 & $\mathrm{U}$ & $10,000 \mathrm{U}$ & 1,000 & $\mathrm{U}$ \\
\hline MW-68C2 & 47.9 & $10,000 \mathrm{U}$ & 5,000 & $\mathrm{U}$ & $10,000 \mathrm{U}$ & 5,000 & $\mathrm{U}$ & 10,000 & $\mathrm{U}$ & 5,000 & $\mathrm{U}$ & $10,000 \mathrm{U}$ & 5,000 & $\mathrm{U}$ \\
\hline MW-68C2 & 49.9 & $10,000 \mathrm{U}$ & 2,500 & $\mathrm{U}$ & $10,000 \mathrm{U}$ & 2,500 & $\mathrm{U}$ & 10,000 & $\mathrm{U}$ & 2,500 & $\mathrm{U}$ & $10,000 \mathrm{U}$ & 2,500 & $\mathrm{U}$ \\
\hline MW-68C2 & 52.0 & $2,500 \mathrm{U}$ & 2,500 & $\mathrm{U}$ & $2,500 \mathrm{U}$ & 2,500 & $\mathrm{U}$ & 2,500 & $\mathrm{U}$ & 2,500 & $\mathrm{U}$ & $2,500 \mathrm{U}$ & 2,500 & $\mathrm{U}$ \\
\hline MW-68C2 & 53.9 & $1,000 \mathrm{U}$ & 2,500 & $\mathrm{U}$ & $1,000 \mathrm{U}$ & 2,500 & $\mathrm{U}$ & 1,000 & $\mathrm{U}$ & 2,500 & $\mathrm{U}$ & $1,000 \mathrm{U}$ & 2,500 & $\mathrm{U}$ \\
\hline MW-68C2 & 55.6 & $500 \mathrm{U}$ & 1,000 & $\mathrm{U}$ & $500 \mathrm{U}$ & 1,000 & $\mathrm{U}$ & 500 & $\mathrm{U}$ & 1,000 & $\mathrm{U}$ & $500 \mathrm{U}$ & 1,000 & $\mathrm{U}$ \\
\hline MW-68C2 & 57.5 & $500 \mathrm{U}$ & 500 & $\mathrm{U}$ & $500 \mathrm{U}$ & 500 & $\mathrm{U}$ & 500 & $\mathrm{U}$ & 500 & $\mathrm{U}$ & $500 \mathrm{U}$ & 500 & $\mathrm{U}$ \\
\hline MW-68C2 & 59.5 & $500 \mathrm{U}$ & 500 & $\mathrm{U}$ & $500 \mathrm{U}$ & 500 & $\mathrm{U}$ & 500 & $\mathrm{U}$ & 500 & $\mathrm{U}$ & $500 \mathrm{U}$ & 500 & $\mathrm{U}$ \\
\hline MW-68C2 & 61.5 & $500 \mathrm{U}$ & 500 & $\mathrm{U}$ & $500 \mathrm{U}$ & 500 & $\mathrm{U}$ & 500 & $\mathrm{U}$ & 500 & $\mathrm{U}$ & $500 \mathrm{U}$ & 500 & $\mathrm{U}$ \\
\hline PW-15 & 25.4 & $4 \mathrm{~J}$ & 2 & $\mathrm{~J}$ & 16 & 9 & & 7 & & & $\mathrm{~J}$ & 75 & 28 & \\
\hline PW-15 & 27.1 & $3 \mathrm{~J}$ & 3 & $\mathrm{~J}$ & 15 & & $\mathrm{~J}$ & & $\mathrm{~J}$ & 5 & $\mathrm{U}$ & 52 & 7 & \\
\hline PW-15 & 28.5 & $100 \mathrm{U}$ & 250 & $\mathrm{U}$ & $100 \mathrm{U}$ & 250 & $\mathrm{U}$ & 100 & $\mathrm{U}$ & 250 & $\mathrm{U}$ & $100 \mathrm{U}$ & 250 & $\mathrm{U}$ \\
\hline PW-15 & 30.2 & $250 \mathrm{U}$ & 500 & $\mathrm{U}$ & $250 \mathrm{U}$ & 500 & $\mathrm{U}$ & 250 & $\mathrm{U}$ & 500 & $\mathrm{U}$ & $250 \mathrm{U}$ & 500 & $\mathrm{U}$ \\
\hline PW-15 & 31.8 & $500 \mathrm{U}$ & 500 & $\mathrm{U}$ & $500 \mathrm{U}$ & 500 & $\mathrm{U}$ & 500 & $\mathrm{U}$ & 500 & $\mathrm{U}$ & $500 \mathrm{U}$ & 500 & $\mathrm{U}$ \\
\hline PW-15 & 33.2 & $500 \mathrm{U}$ & 500 & $\mathrm{U}$ & $500 \mathrm{U}$ & 500 & $\mathrm{U}$ & 500 & $\mathrm{U}$ & 500 & $\mathrm{U}$ & $500 \mathrm{U}$ & 500 & $\mathrm{U}$ \\
\hline PW-55 & 27.1 & $2,500 \mathrm{U}$ & 2,500 & $\mathrm{U}$ & $2,500 \mathrm{U}$ & 2,500 & $\mathrm{U}$ & 2,500 & $\mathrm{U}$ & 2,500 & $\mathrm{U}$ & $2,500 \mathrm{U}$ & 2,500 & $\mathrm{U}$ \\
\hline PW-55 & 28.9 & $2,500 \mathrm{U}$ & 2,500 & $\mathrm{U}$ & $2,500 \mathrm{U}$ & 2,500 & $\mathrm{U}$ & 2,500 & $\mathrm{U}$ & 2,500 & $\mathrm{U}$ & $2,500 \mathrm{U}$ & 2,500 & $\mathrm{U}$ \\
\hline PW-55 & 30.6 & $2,500 \mathrm{U}$ & 2,500 & $\mathrm{U}$ & $2,500 \mathrm{U}$ & 2,500 & $\mathrm{U}$ & 2,500 & $\mathrm{U}$ & 2,500 & $\mathrm{U}$ & $2,500 \mathrm{U}$ & 2,500 & $\mathrm{U}$ \\
\hline
\end{tabular}


Table 6. Concentrations of benzene, ethylbenzene, toluene, and total xylenes in water from diffusion and lowflow sampling, Naval Air Station North Island, California, January 2000-Continued

[ $\mu \mathrm{g} / \mathrm{L}$, micrograms per liter; $\mathrm{U}$, value was below the analytical quantitation limit; J, estimated value; NA, not applicable; *, sample collected by using bladder pump; \#, data from OHM Remediation Services Corporation (2000)

\begin{tabular}{|c|c|c|c|c|c|c|c|c|c|}
\hline \multirow[t]{2}{*}{ Well } & \multirow{2}{*}{$\begin{array}{l}\text { Depth } \\
\text { (feet) }\end{array}$} & \multicolumn{2}{|c|}{$\begin{array}{c}\text { Benzene } \\
(\mu \mathrm{g} / \mathrm{L})\end{array}$} & \multicolumn{2}{|c|}{$\begin{array}{c}\text { Ethylbenzene } \\
(\mu \mathrm{g} / \mathrm{L})\end{array}$} & \multicolumn{2}{|c|}{$\begin{array}{c}\text { Toluene } \\
(\mu \mathrm{g} / \mathrm{L})\end{array}$} & \multicolumn{2}{|c|}{$\begin{array}{c}\text { Total xylenes } \\
(\mu \mathrm{g} / \mathrm{L})\end{array}$} \\
\hline & & Diffusion & Low flow & Diffusion & Low flow & Diffusion & Low flow & Diffusion & Low flow \\
\hline PW-55 & 31.9 & $2,500 \mathrm{U}$ & $2,500 \mathrm{U}$ & $2,500 \mathrm{U}$ & $2,500 \mathrm{U}$ & $2,500 \mathrm{U}$ & $2,500 \mathrm{U}$ & $2,500 \mathrm{U}$ & $2,500 \mathrm{U}$ \\
\hline PW-55 & 33.1 & $2,500 \mathrm{U}$ & $2,500 \mathrm{U}$ & $2,500 \mathrm{U}$ & $2,500 \mathrm{U}$ & $2,500 \mathrm{U}$ & $2,500 \mathrm{U}$ & $2,500 \mathrm{U}$ & $2,500 \mathrm{U}$ \\
\hline PW-66 & 25.5 & $1,000 \mathrm{U}$ & $500 \mathrm{U}$ & $1,000 \mathrm{U}$ & $500 \mathrm{U}$ & $1,000 \mathrm{U}$ & $500 \mathrm{U}$ & $1,000 \mathrm{U}$ & $500 \mathrm{U}$ \\
\hline PW-66 & 27.3 & $500 \mathrm{U}$ & $500 \mathrm{U}$ & $500 \mathrm{U}$ & $500 \mathrm{U}$ & $500 \mathrm{U}$ & $500 \mathrm{U}$ & $500 \mathrm{U}$ & $500 \mathrm{U}$ \\
\hline PW-66 & 29.1 & $50 \mathrm{U}$ & $500 \mathrm{U}$ & $50 \mathrm{U}$ & $500 \mathrm{U}$ & $50 \mathrm{U}$ & $500 \mathrm{U}$ & $50 \mathrm{U}$ & $500 \mathrm{U}$ \\
\hline PW-66 & 30.8 & $25 \mathrm{U}$ & $500 \mathrm{U}$ & $25 \mathrm{U}$ & $500 \mathrm{U}$ & $25 \mathrm{U}$ & $500 \mathrm{U}$ & $25 \mathrm{U}$ & $500 \mathrm{U}$ \\
\hline PW-66 & 32.3 & $5 \mathrm{U}$ & $500 \mathrm{U}$ & $5 \mathrm{U}$ & $500 \mathrm{U}$ & $5 \mathrm{U}$ & $500 \mathrm{U}$ & $5 \mathrm{U}$ & $500 \mathrm{U}$ \\
\hline PW-66 & 33.9 & $5 \mathrm{U}$ & $500 \mathrm{U}$ & $5 \mathrm{U}$ & $500 \mathrm{U}$ & $5 \mathrm{U}$ & $500 \mathrm{U}$ & $5 \mathrm{U}$ & $500 \mathrm{U}$ \\
\hline S2-MW-6A & 6.5 & $5 \mathrm{U}$ & $5 \mathrm{U}$ & $5 \mathrm{U}$ & $5 \mathrm{U}$ & $5 \mathrm{U}$ & $5 \mathrm{U}$ & $5 \mathrm{U}$ & $5 \mathrm{U}$ \\
\hline S2-MW-6A & 7.9 & $5 \mathrm{U}$ & $5 \mathrm{U}$ & $5 \mathrm{U}$ & $5 \mathrm{U}$ & $5 \mathrm{U}$ & $5 \mathrm{U}$ & $5 \mathrm{U}$ & $5 \mathrm{U}$ \\
\hline S2-MW-6A & 9.2 & $5 \mathrm{U}$ & $5 \mathrm{U}$ & $5 \mathrm{U}$ & $5 \mathrm{U}$ & $5 \mathrm{U}$ & $5 \mathrm{U}$ & $5 \mathrm{U}$ & $5 \mathrm{U}$ \\
\hline S2-MW-6A & 10.6 & $5 \mathrm{U}$ & $5 \mathrm{U}$ & $5 \mathrm{U}$ & $5 \mathrm{U}$ & $5 \mathrm{U}$ & $5 \mathrm{U}$ & $5 \mathrm{U}$ & $5 \mathrm{U}$ \\
\hline S2-MW-6A & 12.0 & $5 \mathrm{U}$ & $5 \mathrm{U}$ & $5 \mathrm{U}$ & $5 \mathrm{U}$ & $5 \mathrm{U}$ & $5 \mathrm{U}$ & $5 \mathrm{U}$ & $5 \mathrm{U}$ \\
\hline S2-MW-6A & 13.3 & $5 \mathrm{U}$ & $5 \mathrm{U}$ & $5 \mathrm{U}$ & $5 \mathrm{U}$ & $5 \mathrm{U}$ & $5 \mathrm{U}$ & $5 \mathrm{U}$ & $5 \mathrm{U}$ \\
\hline S2-MW-6A & 14.7 & $5 \mathrm{U}$ & $5 \mathrm{U}$ & $5 \mathrm{U}$ & $5 \mathrm{U}$ & $5 \mathrm{U}$ & $5 \mathrm{U}$ & $5 \mathrm{U}$ & $5 \mathrm{U}$ \\
\hline S2-MW-6A & 16.1 & $5 \mathrm{U}$ & $5 \mathrm{U}$ & $5 \mathrm{U}$ & $5 \mathrm{U}$ & $5 \mathrm{U}$ & $5 \mathrm{U}$ & $5 \mathrm{U}$ & $5 \mathrm{U}$ \\
\hline S2-MW-6A & 17.5 & $5 \mathrm{U}$ & $5 \mathrm{U}$ & $5 \mathrm{U}$ & $5 \mathrm{U}$ & $5 \mathrm{U}$ & $5 \mathrm{U}$ & $5 \mathrm{U}$ & $5 \mathrm{U}$ \\
\hline S2-MW-6A & 19.0 & $5 \mathrm{U}$ & $5 \mathrm{U}$ & $5 \mathrm{U}$ & $5 \mathrm{U}$ & $5 \mathrm{U}$ & $5 \mathrm{U}$ & $5 \mathrm{U}$ & $5 \mathrm{U}$ \\
\hline
\end{tabular}

20 percent, the concentrations obtained using the diffusion samplers were slightly higher than those concentrations obtained using the bladder pump. The concentrations obtained using the diffusion samplers in well MW-13B were slightly higher, but similar to the concentrations obtained using the peristaltic pump (fig. 3). Concentrations of toluene and total xylenes were present in water obtained from both the diffusion samplers and the peristaltic pump (fig. 3); toluene and total xylenes were not detectable (less than $5 \mu \mathrm{g} / \mathrm{L}$ ) in water from the bladder pump. The data suggest that the diffusion samplers performed equally well with the bladder pump in wells MW-13A and MW-13B for $c$ DCE. The higher concentrations of vinyl chloride, toluene, and total xylenes in water from the diffusion samplers relative to water from the bladder pump indicate that the diffusion samplers obtained more discrete samples from these wells; however, disturbing the well water by using the peristaltic pump and removing the diffusion samplers prior to sampling with the bladder pump may have induced mixing and affected the quality of the water sampled by the bladder pump.

\section{Comparison of Diffusion-Sampler Results to Peristaltic-Pump Results}

The remaining comparisons between diffusion-sampler and low-flow sampler methods utilized multiple diffusion-sampling and low-flow sampling points within screened intervals. At most depths, low-flow sampling was conducted by using peristaltic pumps. In contrast to bladder pumps, using peristaltic pumps in some wells potentially could cause degassing of samples during recovery, which could result in underestimating actual VOC concentrations. Thus, VOC concentrations in water obtained using peristaltic 
Table 7. Comparison of concentrations of selected volatile organic compounds in water from a diffusion sampler and in water from low-flow purging using a bladder pump at the same depth, Naval Air Station North Island, California, January 2000

[*, average percent difference; -, concentration measured in diffusion sampler was lower than concentration measured in low-flow sample]

\begin{tabular}{|c|c|c|c|c|c|}
\hline \multirow[b]{2}{*}{ Constituent } & \multicolumn{2}{|c|}{ Diffusion samples } & \multicolumn{2}{|c|}{ Low-flow bladder-pump samples } & \multirow[b]{2}{*}{$\begin{array}{l}\text { Percent } \\
\text { difference }\end{array}$} \\
\hline & $\begin{array}{l}\text { Depth, in feet } \\
\text { below land } \\
\text { surface }\end{array}$ & $\begin{array}{c}\text { Concentration, } \\
\text { in micrograms } \\
\text { per liter }\end{array}$ & $\begin{array}{l}\text { Depth, in feet } \\
\text { below land } \\
\text { surface }\end{array}$ & $\begin{array}{c}\text { Concentration, } \\
\text { in micrograms } \\
\text { per liter }\end{array}$ & \\
\hline \multicolumn{6}{|c|}{ Well MW-9 } \\
\hline $\begin{array}{l}\text { 1,1-Dichloroethene } \\
\text { (1,1-DCE) }\end{array}$ & 31 & 4,000 & 31 & 3,500 & 2.0 \\
\hline Tetrachloroethene (PCE) & 31 & 260 & 31 & 330 & -21.0 \\
\hline Trichloroethene (TCE) & 31 & 3,300 & 31 & 3,200 & 3.0 \\
\hline \multicolumn{6}{|c|}{ Well MW-5 } \\
\hline Trichloroethene (TCE) & 55.75 & 360 & 55.75 & 280,320 & $17 *$ \\
\hline \multicolumn{6}{|c|}{ Well MW-12 } \\
\hline $\begin{array}{l}\text { cis-1,2-Dichloroethene } \\
\quad \text { (cDCE) }\end{array}$ & 35.1 & 100 & 35.1 & 450 & -78 \\
\hline Trichloroethene (TCE) & 35.1 & 1,800 & 35.1 & 2,100 & -14 \\
\hline \multicolumn{6}{|c|}{ Well MW-13A } \\
\hline $\begin{array}{l}\text { cis-1,2-Dichloroethene } \\
\quad \text { (cDCE) }\end{array}$ & $10.9-12.4$ & $46-74$ & 12 & 61 & Within range \\
\hline Vinyl chloride & $10.9-12.4$ & $5-8$ & 12 & 7.4 & Within range \\
\hline \multicolumn{6}{|c|}{ Well MW-13B } \\
\hline $\begin{array}{l}\text { cis-1,2-Dichloroethene } \\
\quad \text { (cDCE) }\end{array}$ & $24.85-26.15$ & $3,100-2,600$ & 26 & 3,100 & Within range \\
\hline Toluene & $24.85-26.15$ & 9 & 26 & $<5$ & Not applicable \\
\hline Total xylenes & $24.85-26.15$ & $111-110$ & 26 & $<5$ & Not applicable \\
\hline Vinyl chloride & $24.85-26.15$ & $1,900-2,000$ & 26 & 1,600 & $18^{*}$ \\
\hline
\end{tabular}

pumps may be representative of concentrations in ground water at some wells but may underestimate actual concentrations in ground water at other wells. Moreover, when multiple depths within a screened interval are purged using low-flow methods, there is a potential for each low-flow sampling event to disturb the equilibrated water column. If the pumping rate during low-flow sampling is low enough to prevent drawdown in the well, then all of the pumped water is replaced by ground water from the aquifer; however, the zone of influence contributing water to the well may not be adjacent to the pump. Thus, in a chemically stratified screened interval where multiple depth intervals are sequentially sampled, water entering the well screen from early low-flow samplings may influence concentrations obtained in later samplings as a result of vertical transport and mixing in the well screen. Despite these uncertainties, the use of multiple-level low-flow sampling methods using peristaltic pumps sometimes can provide an estimate of contaminant vertical distribution in the screened interval, which can be used as a comparison for the diffusion samplers.

In most of the observation wells, the vertical concentration gradients obtained using the diffusionsampler and low-flow sampler methods were similar. However, in several cases, the concentrations in water obtained by using the peristaltic pump were lower than the concentrations in water obtained by using the diffusion samplers (figs. 4, 5, and 6). An example of this is TCE concentrations measured in water from wells MW-68A, MW-68B, and MW-68C. TCE concentrations were approximately 43 to 73 percent lower in water samples collected by using low-flow sampling methods and peristaltic pumps than in samples collected by the diffusion samplers. This substantial difference in concentrations between the two methods is expected if VOCs were lost by degassing as a result of 

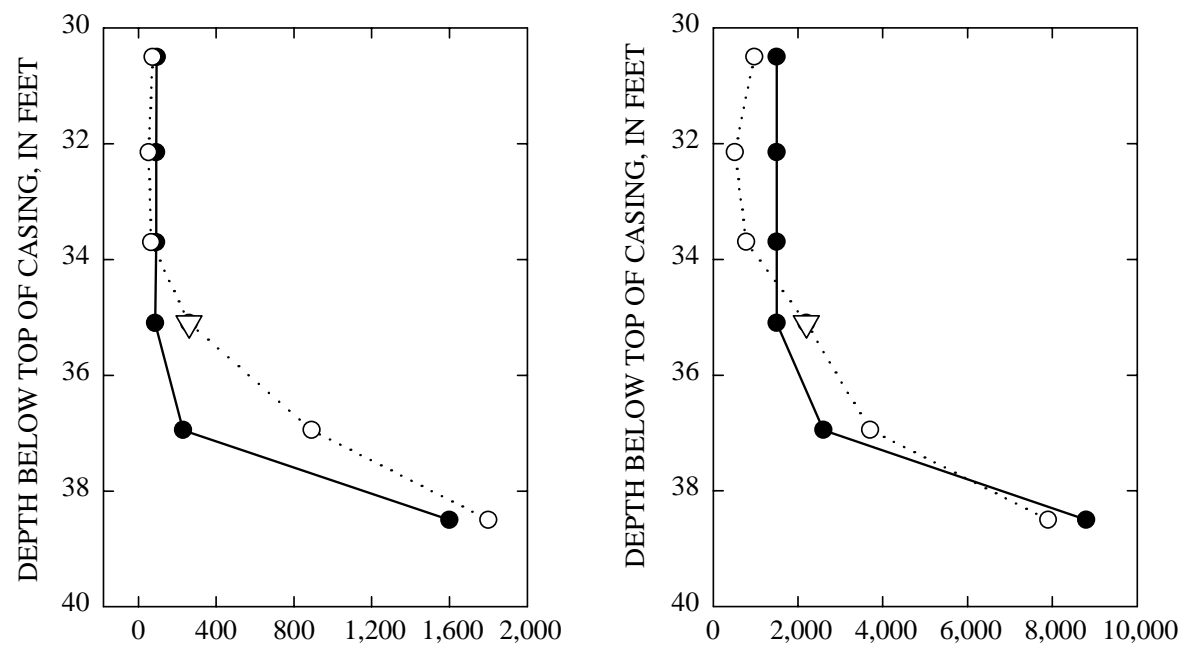

1,1-DICHLOROETHANE CONCENTRATION, IN MICROGRAMS PER LITER

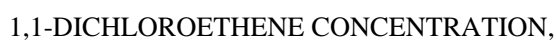
IN MICROGRAMS PER LITER
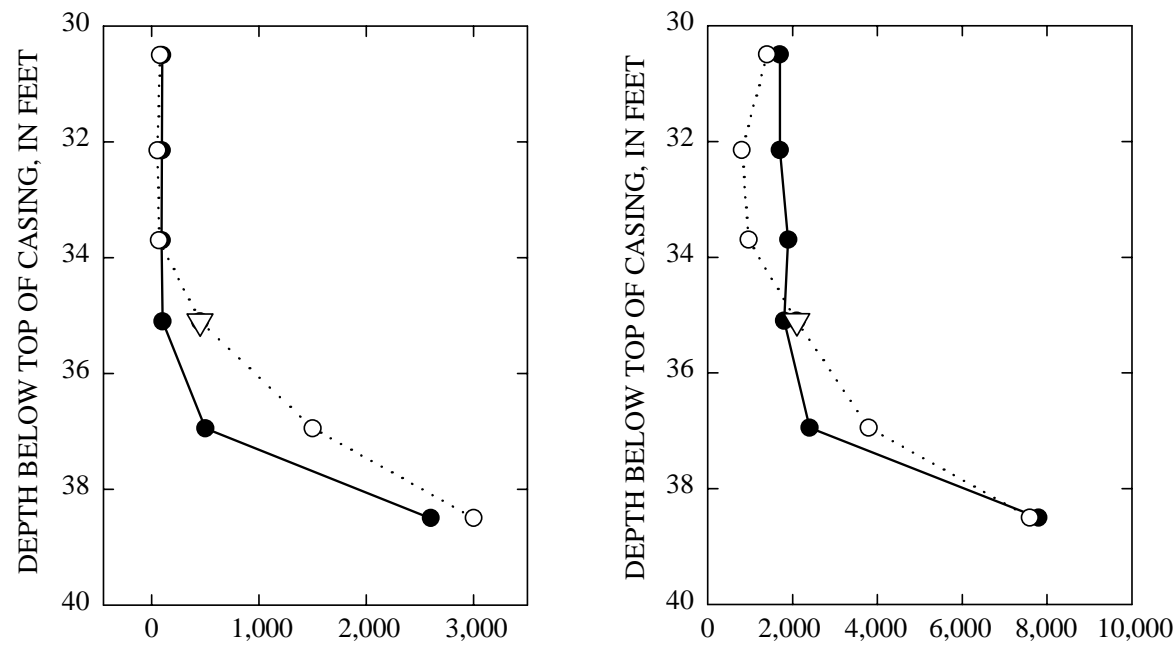

cis-1,2-DICHLOROETHENE CONCENTRATION, IN MICROGRAMS PER LITER

TRICHLOROETHENE CONCENTRATION, IN MICROGRAMS PER LITER

\author{
EXPLANATION \\ $\multimap$ DIFFUSION SAMPLE \\ … LOW-FLOW PERISTALTIC- \\ PUMP SAMPLE \\ $\nabla \quad$ LOW-FLOW BLADDER-PUMP \\ SAMPLE
}

Figure 2. Comparison of diffusion and low-flow samples in ground water at well MW-12, Naval Air Station North Island, California, January 2000. 

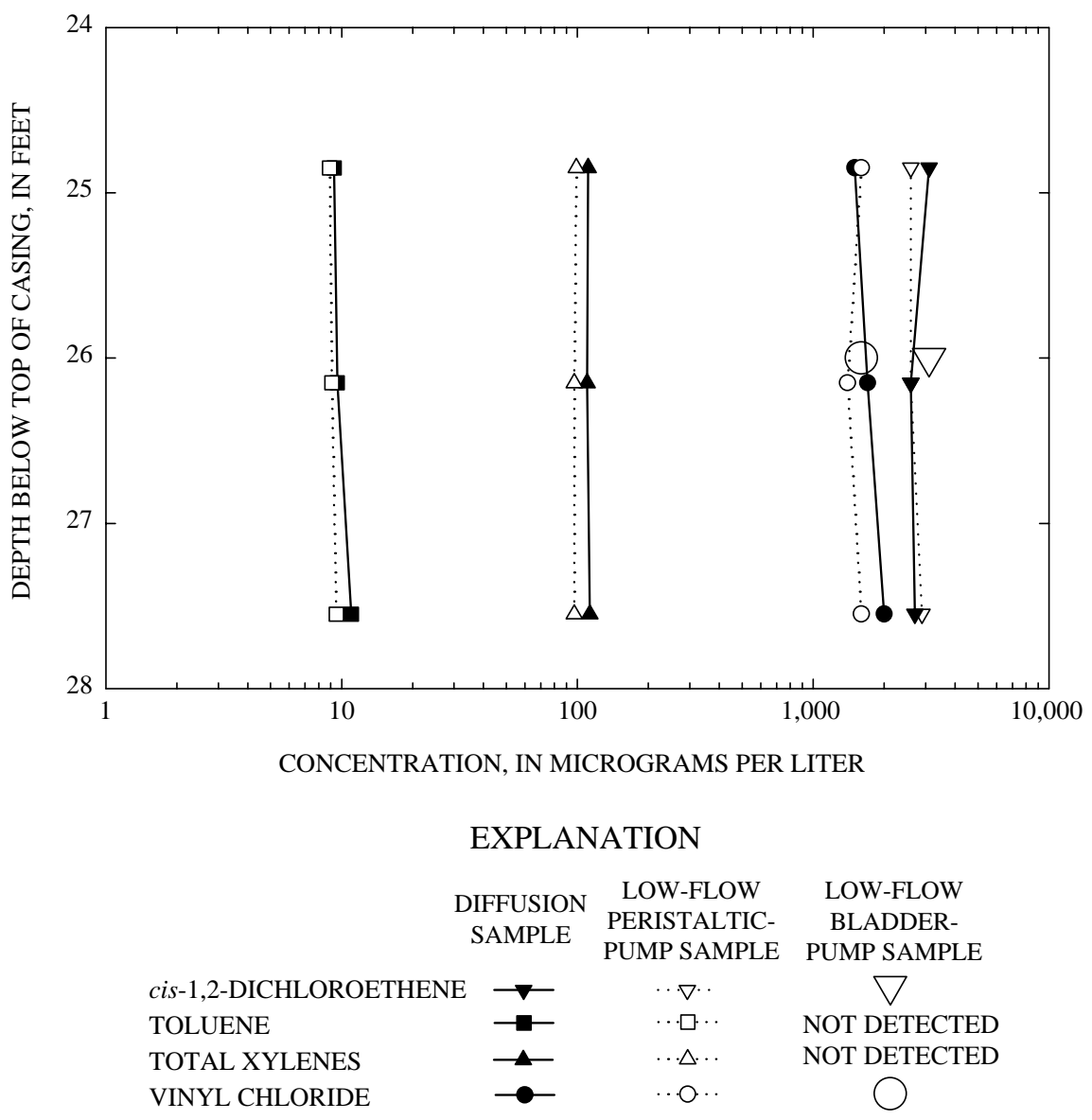

Figure 3. Comparison of diffusion and low-flow samples in ground water at well MW-13B, Naval Air Station North Island, California, January 2000.

using peristaltic pumps or if mixing in the well screens occurred during pumping. The vertical concentration distribution between the two methods implies that the VOC concentrations measured in water from diffusion samplers reflected the vertical distribution of contaminants in the aquifer adjacent to the screened interval more accurately than the peristaltic-pump sampling.

Further comparison of TCE concentration data from the two sampling methods indicates that diffusion sampling provides a point sample, whereas sequential low-flow sampling of multiple horizons within a single well screen can induce mixing. In general, the vertical sequence of low-flow sampling in the wells began with the shallowest depth interval and ended with the deepest interval. In well PW-66, TCE data show that concentrations in water collected with a diffusion sampler were highest in the shallowest sampled depth, and then decreased sharply over the 5-ft depth interval below this shallowest depth (fig. 7).
Although the highest TCE concentration obtained by low-flow sampling also was at the shallowest horizon, it was approximately 24 percent lower than the concentration obtained from the corresponding diffusion sampler, and the vertical stratification was less sharply defined. These data suggest that as low-flow sampling with a peristaltic pump progressed vertically downward, the pumping gradually mixed the TCE-contaminated water from the shallowest sampling depth with water from deeper intervals, thus obscuring the original contaminant stratification (fig. 7).

A similar effect can be seen in the data from wells MW-12 and PW-15 (figs. 2 and 8). At these wells, the shallowest interval was relatively uncontaminated. The comparison between diffusion samples and low-flow samples at this shallowest depth showed a relatively close match between $c$ DCE and TCE concentrations. However, as sampling progressed vertically downward toward the interface of the 


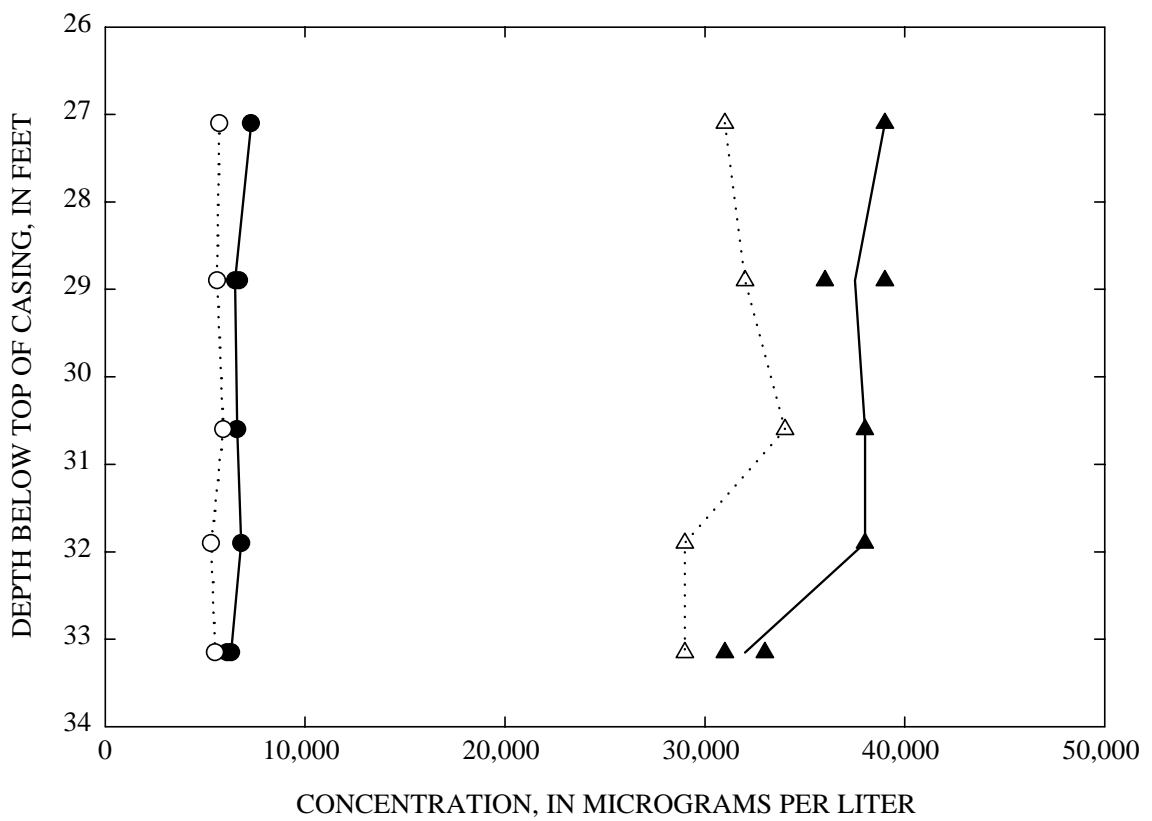

EXPLANATION

$\begin{array}{llc} & \begin{array}{l}\text { DIFFUSION } \\ \text { SAMPLE }\end{array} & \begin{array}{c}\text { LOW-FLOW } \\ \text { SAMPLE }\end{array} \\ \text { cis-1,2-DICHLOROETHENE } & \bullet & \cdots . . \\ \text { TRICHLOROETHENE } & - & \cdots .\end{array}$

Figure 4. Comparison of diffusion and low-flow samples in ground water at well PW-55, Naval Air Station North Island, California, January 2000.

stratified contamination, the low-flow sample concentrations generally increased higher than the diffusionsample concentrations, which is to be expected if the zone of influence for the low-flow pumping captured the more contaminated ground water in the well. In general, the data suggest that diffusion sampling provides a more precise delineation of the contaminant stratification within the screened interval than lowflow sampling.

Insight into the use of diffusion samplers in a chemically stratified screened interval can be observed in the data from wells at the MW-68 cluster (figs. 6D and $6 \mathrm{E})$. Unlike the other wells, two peristaltic pumps were used to low-flow sample well MW-68C2. Starting simultaneously from both the uppermost and the lowermost sample depths, sampling progressed sequentially toward the center of the $25-\mathrm{ft}$ screened interval. Results from both the diffusion samples and the low-flow samples showed that the uppermost and lowermost parts of the screened interval were relatively uncontaminated. Concentration data from the diffusion samples show that substantially higher TCE concentrations occurred between depths of approximately 40 to $50 \mathrm{ft}$, with a sharp peak at about $42 \mathrm{ft}$ (fig. 6D). Thus, the first water pulled into the well screen from both ends of the screen was relatively uncontaminated. As the low-flow sampling progressed toward the center of the screened interval, the correlation between concentrations obtained from the diffusion samples began to differ substantially from those obtained by low-flow sampling (fig. 6D). Between the depths of approximately 40 to $50 \mathrm{ft}$, TCE concentrations from low-flow sampling were approximately 47 to 84 percent lower than TCE concentrations from diffusion samplers; additionally, the low-flow sampling data did not indicate a TCE peak concentration at a depth of $42 \mathrm{ft}$ as shown by the diffusion sampling data. A probable explanation for the concentration differences between the two methods is that initially, relatively uncontaminated water was pumped into the screened interval, thus mixing the ground water in the well and diluting concentrations of TCE. As a result, 

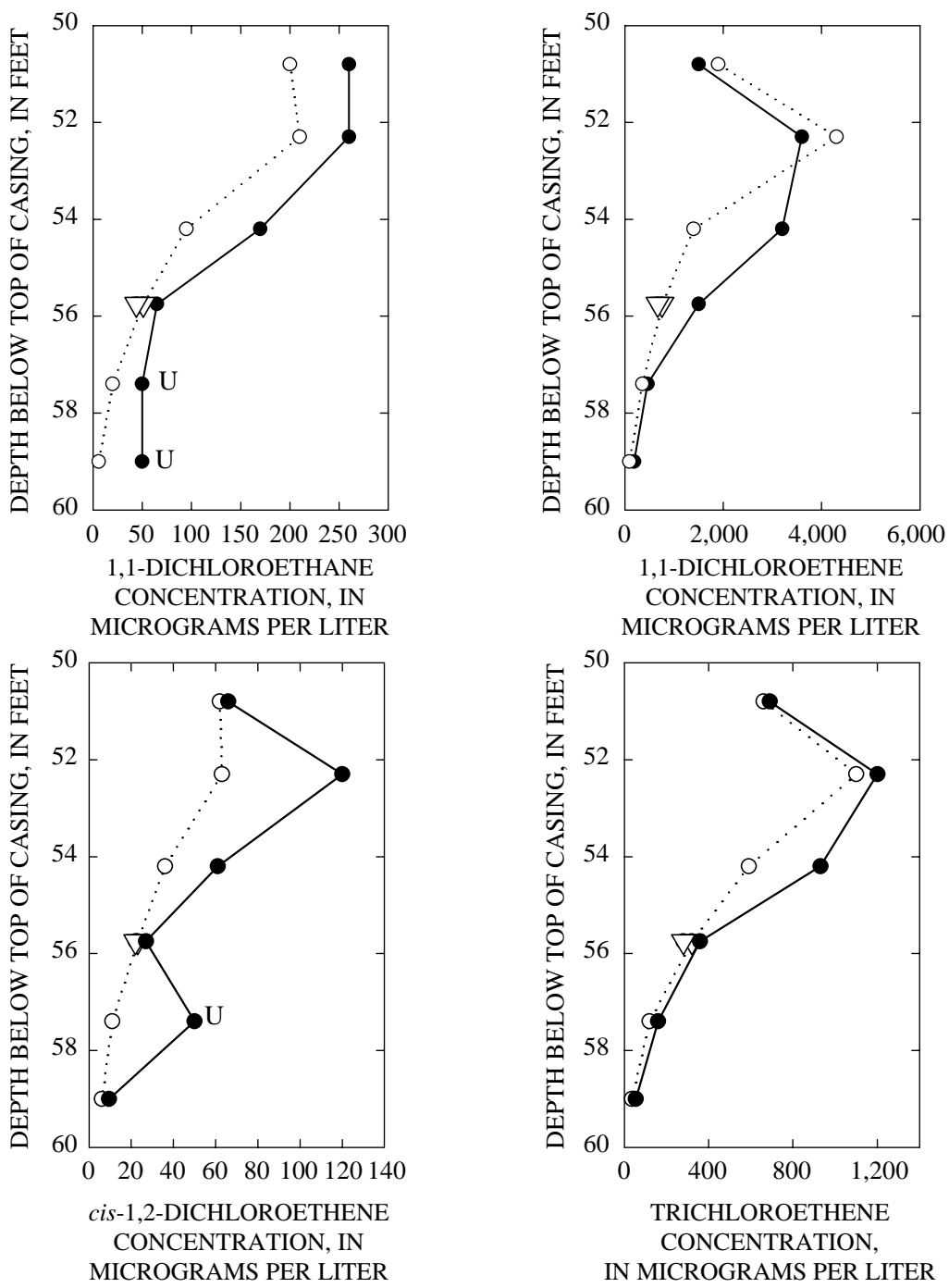

$$
\begin{aligned}
& \text { EXPLANATION } \\
& \bullet \text { DIFFUSION SAMPLE } \\
& \text {. o . . LOW-FLOW PERISTALTIC- } \\
& \text { PUMP SAMPLE } \\
& \nabla \quad \text { LOW-FLOW BLADDER-PUMP } \\
& \text { SAMPLE } \\
& \text { U VALUE WAS BELOW THE ANALYTICAL } \\
& \text { QUANTITATION LIMIT }
\end{aligned}
$$

Figure 5. Comparison of diffusion and low-flow samples in ground water at well MW-5, Naval Air Station North Island, California, January 2000. 

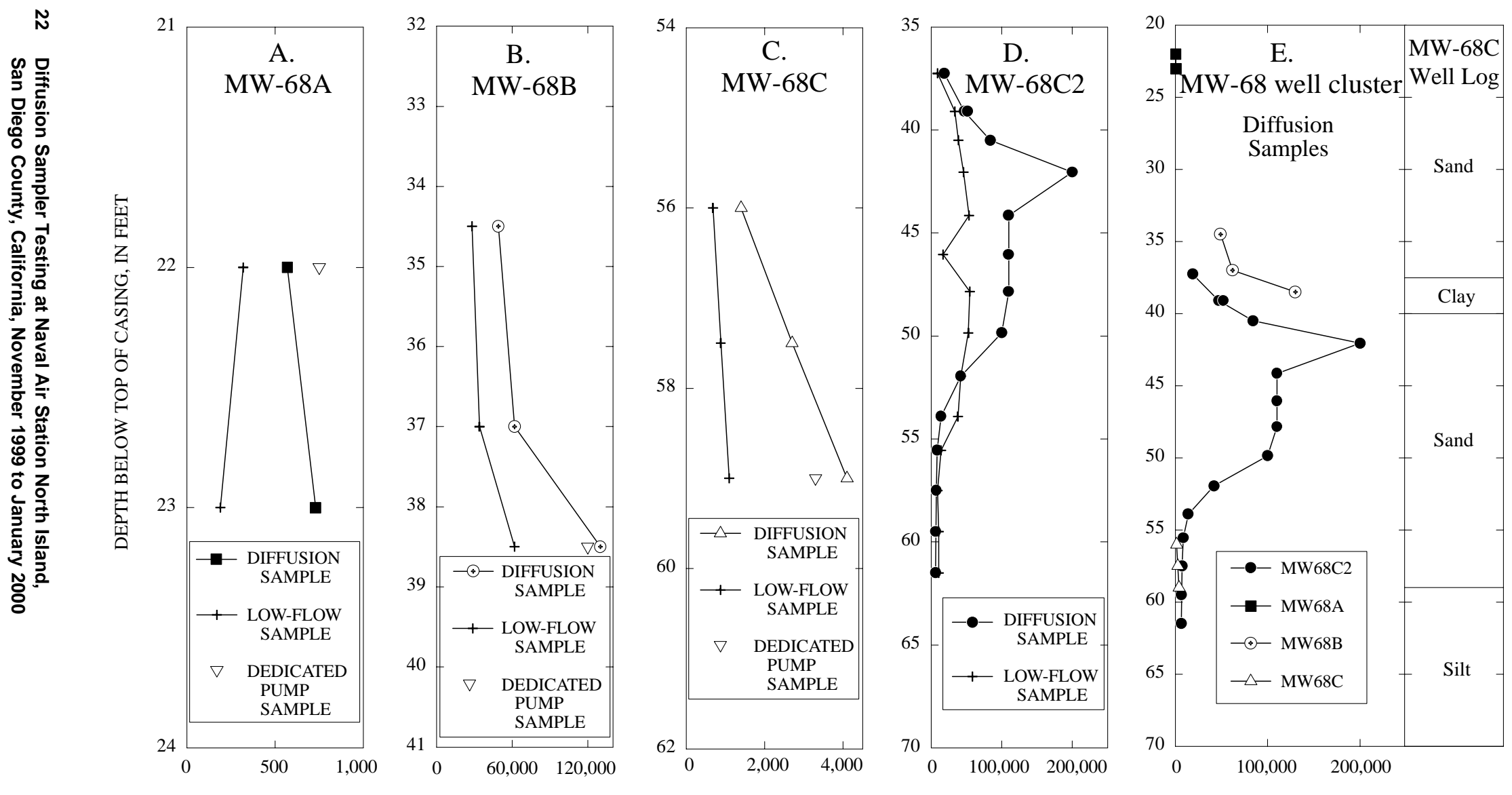

Figure 6. Comparison of diffusion and low-flow samples in ground water at wells (A) MW-68A, (B) MW-68B, (C) MW-68C, and (D) MW-68C2, and (E) comparison of diffusion samples from multiple wells to geologic log of well MW-68C, Naval Air Station North Island, California, January 2000. 


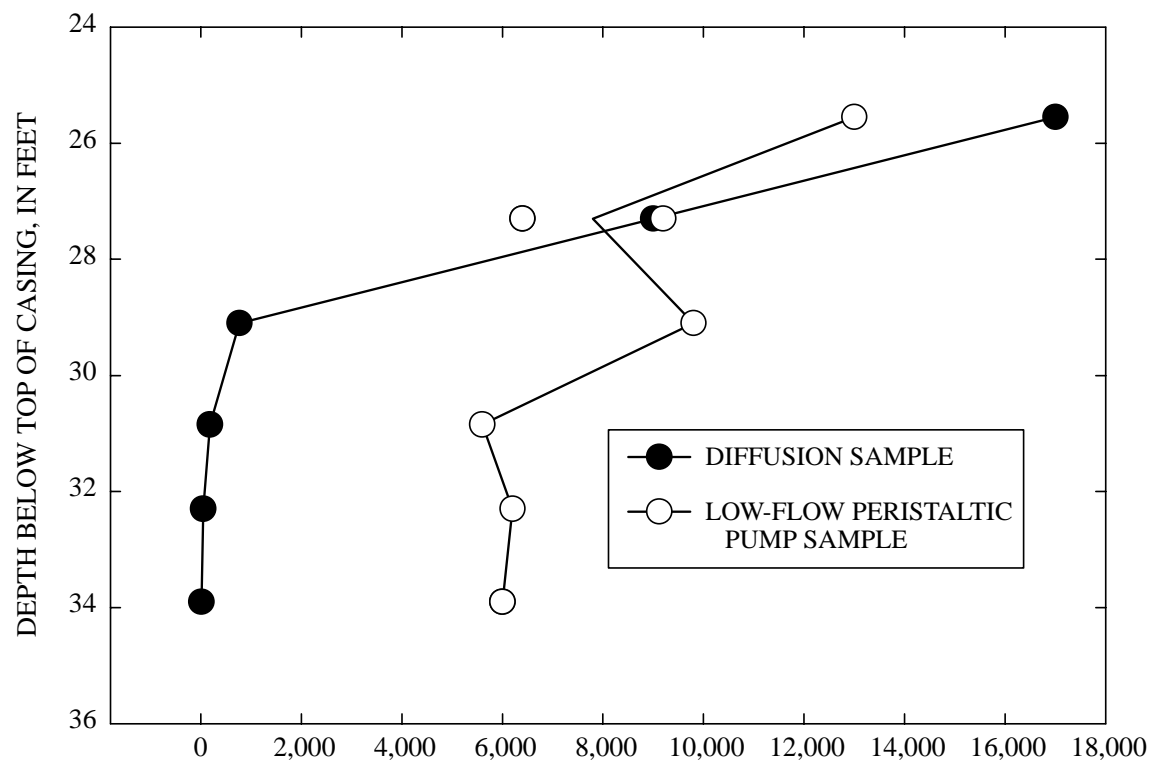

TRICHLOROETHENE CONCENTRATION, IN MICROGRAMS PER LITER

Figure 7. Comparison of trichloroethene concentrations in diffusion and low-flow samples in ground water at well PW-66, Naval Air Station North Island, California 2000.
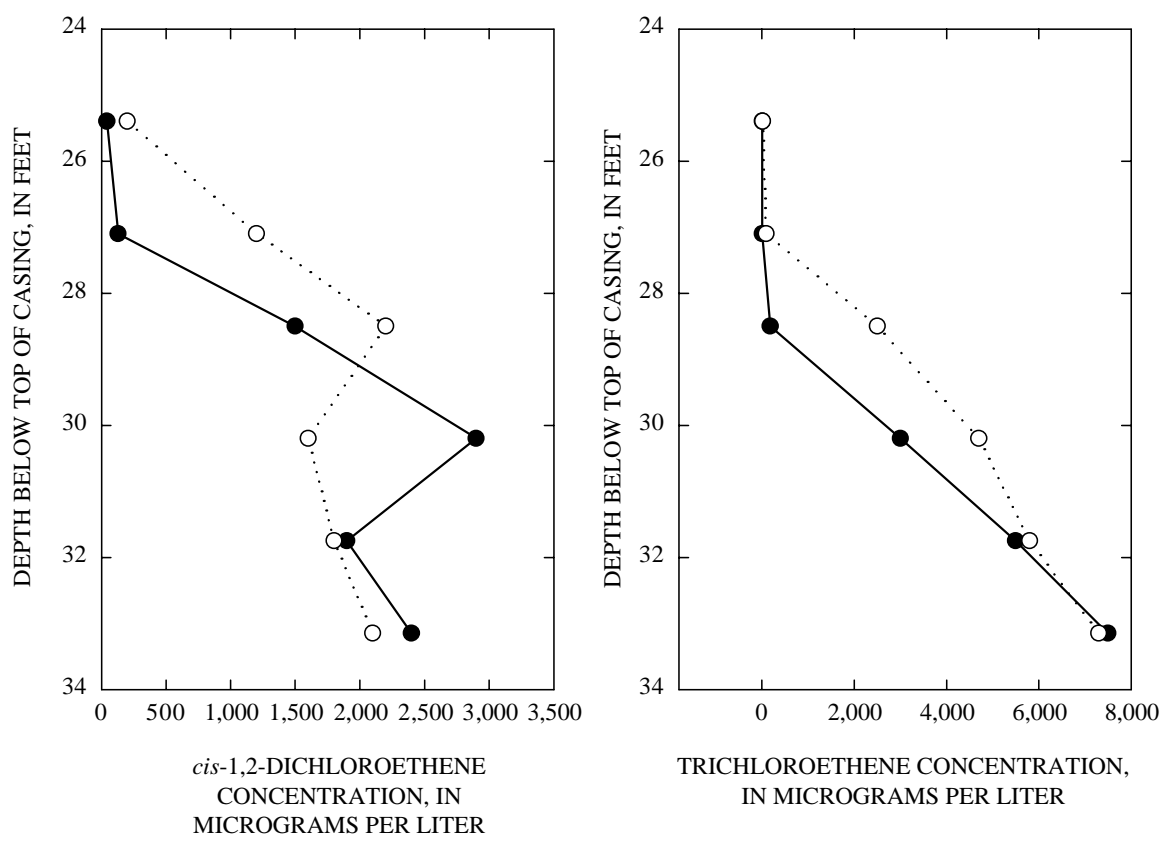

Figure 8. Comparison of diffusion and low-flow samples in ground water at well PW-15, Naval Air Station North Island, California, January 2000. 
TCE concentrations were lowered in ground water collected from subsequently sampled depths. Additional VOC losses by degassing during the use of peristaltic pumps probably resulted in further concentration differences between the two sampling methods.

TCE concentration data in diffusion samplers collected from wells MW-68B and MW-68C and concentration data in diffusion samplers collected from adjacent well MW-68C2 support the vertical distribution indicated by the diffusion samplers in well MW-68C2 (figs. 6B, 6C, and 6D). Diffusion samplers from well MW-68C2 indicate that the lowest concentrations in the screened interval are below a depth of approximately $55 \mathrm{ft}$, and the detected concentrations are similar to those from the same depth in the adjacent well MW-68C (fig. 6E).

Similarly, diffusion samplers from wells MW-68C2 and MW-68B both indicate TCE concentrations increasing with depth between approximately 35 and $40 \mathrm{ft}$ (fig. 6E). The TCE concentrations in diffusion samples from well MW-68B are higher than those from the corresponding depth in well MW-68C2 (fig. 6E). The reasons for the concentration difference between wells MW-68C2 and MW-68B are not known; however, two explanations can be postulated. One explanation is that the contaminant concentrations in well MW-68C2 may have been shifted downward as a result of a vertical hydraulic gradient within the well. Water-level measurements are not shown for well MW-68C2 because they would reflect only compositing across the screened interval; however, evidence for such a hydraulic gradient can be seen in the water-level data for wells MW-68B and MW-68C. The water level in well MW-68B is $0.34 \mathrm{ft}$ higher than the water level in well MW-68C, indicating a net downward hydraulic gradient between the two depths (table 1). Water levels remeasured in March 2000 confirmed the hydraulic gradient. Because well MW-68C2 is only about $5 \mathrm{ft}$ from wells MW-68B and MW-68C, and because the screened interval of well MW-68C2 hydraulically connects the depths sampled by wells MW-68B and MW-68C, the probability is high that there also is a downward hydraulic gradient within well MW-68C2. An alternative explanation is that lithologic heterogeneities in the screened zone place the contamination at slightly different depths in different wells. Evidence for such heterogeneity is the clay layer at a depth of 37.5 to $40 \mathrm{ft}$ in well MW-68C (driller's log, Richard Wong, OHM Remediation, written commun., 2000). Despite the uncertainty regarding concentration differences between wells, the diffusion samplers appear to have been successful in approximately locating the zone of highest concentrations between the depths of 37 to $52 \mathrm{ft}$ (fig. 6E).

VOC concentrations in water collected from well MW-13A varied less and generally were lower for peristaltic pump sampling compared to diffusion sampling (fig. 9). Following low-flow sampling using a peristaltic pump, well MW-13A was immediately resampled by low-flow sampling using a bladder pump. Although subject to the same mixing potential as the peristaltic pump, the bladder pump has less potential for volatilization loss than the peristaltic pump, and thus, probably provides a more representative sample than the peristaltic pump. The concentrations of $c$ DCE and TCE in water obtained using lowflow sampling methods with a bladder pump approximated the average of concentrations obtained in water from the diffusion samplers directly above and below the bladder pump (fig. 8). These findings suggest that data obtained by using the diffusion samplers provided depth-specific VOC concentrations while the data from low-flow sampling represented a mixing of waters in well MW-13A.

In well MW-10, low-flow peristaltic-pump sampling detected low concentrations ( $30 \mu \mathrm{g} / \mathrm{L}$ or less) of TCE, whereas diffusion sampling detected none (table 5). This difference in concentrations is unusual because the potential for volatilization loss using the peristaltic pump usually results in underestimating ambient concentrations, while diffusion samplers are capable of producing representative samples even at low (less than $20 \mu \mathrm{g} / \mathrm{L}$ ) concentrations. According to historical data (OHM Remediation Services Corporation, 2000), TCE has never previously been detected in well MW-10 (sampling dates July 1998, March 1999, June 1999, and September 1999). Furthermore, a resampling of the well using low-flow methodology at multiple horizons in February 2000 also showed that TCE was not present. Thus, it seems that the diffusion samplers accurately reflected VOC concentrations in ground water; the source of low TCE concentrations found in water obtained from low-flow, peristalticpump sampling is unknown, but may represent a cross-contamination source not related to local ground water.

Wells S2-MW-06A and MW-13C contained no detectable VOCs (less than $5 \mu \mathrm{g} / \mathrm{L}$ ) in water from either the diffusion samples or from the low-flow samples. Thus, the construction materials used in the 

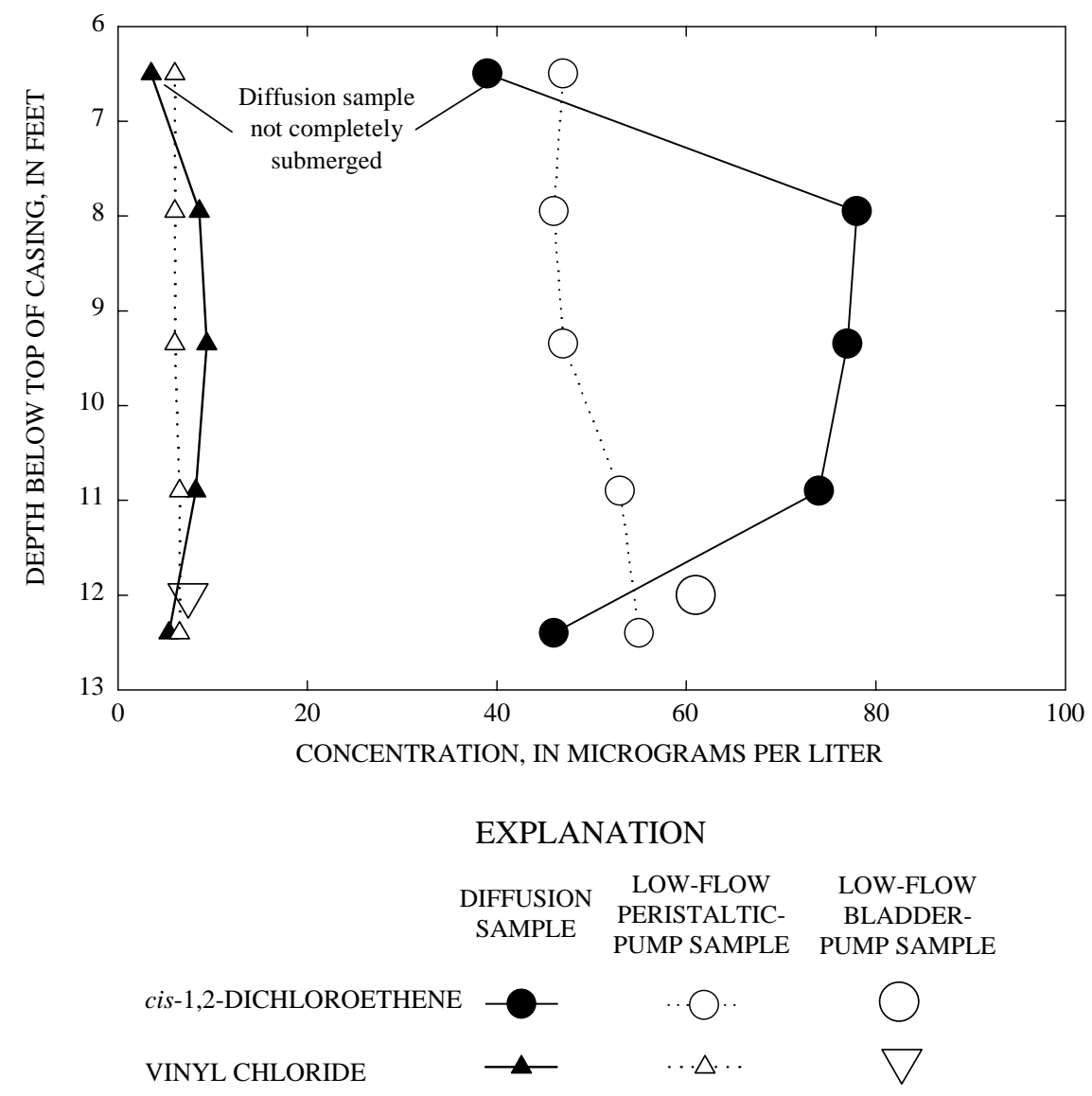

Figure 9. Comparison of diffusion and low-flow samples in ground water well MW-13A, Naval Air Station North Island California, January 2000.

diffusion samplers did not contribute contaminants to the water.

\section{Diffusion Samplers in Free-Phase Fuel}

The diffusion samplers deployed in buckets containing free-phase JP-5 and Stoddard solution from wells MW-11 and PW-17 did not show evidence of structural integrity loss during the 2 months of equilibration. The VOCs detected in the free-phase fuel also were detected in the water from the diffusion samplers (table 8). The VOC concentrations in water from the diffusion samplers were lower than the VOC concentrations in the fuel; however, this is to be expected because the first is an aqueous solution and the second is an organic solvent concentration. The diffusion samplers provided an alternative method for showing that the free-phase fuel in ground water from well MW-11 also contained TCE (table 5).

\section{Contaminant Stratification in Well Screens}

The data from this investigation show that substantial stratification of VOCs can be present within a 10-ft well screen. At four observation wells (MW-12, MW-5, PW-66, and PW-15), the data showed a sharp layering of VOCs within the screened interval (figs. 2, 5,7 , and 8). The diffusion-sampler data show that the vertical change in TCE concentrations over a distance of about $5 \mathrm{ft}$ was approximately $17,500 \mu \mathrm{g} / \mathrm{L}$ in well PW-66, approximately 7,300 $\mu \mathrm{g} / \mathrm{L}$ in well PW-15, and approximately $5,900 \mu \mathrm{g} / \mathrm{L}$ in well MW-12. At well MW-5, the 1,1-DCE concentration changed by $3,410 \mu \mathrm{g} / \mathrm{L}$, and the TCE concentration changed by 
Table 8. Concentrations of selected volatile organic compounds in free-phase jet fuel (JP-5) removed from ground water and in water from diffusion samplers deployed in a bucket containing the free-phase fuel, Naval Air Station North Island, California, January 2000

[ $\mu \mathrm{g} / \mathrm{L}$, micrograms per liter; J, estimated value; $\mathrm{U}$, value was below the analytical quantitation limit]

\begin{tabular}{|c|c|c|c|c|c|c|c|c|c|c|}
\hline \multirow{2}{*}{$\begin{array}{l}\text { Sample } \\
\text { source }\end{array}$} & \multicolumn{2}{|c|}{$\begin{array}{c}\text { 1,1-Dichloroethane } \\
(\mu \mathrm{g} / \mathrm{L})\end{array}$} & \multicolumn{2}{|c|}{$\begin{array}{c}\text { 1,1-Dichloroethene } \\
(\mu \mathrm{g} / \mathrm{L})\end{array}$} & \multicolumn{2}{|c|}{$\begin{array}{c}\text { cis-1,2-Dichloroethene } \\
(\mu \mathrm{g} / \mathrm{L})\end{array}$} & \multicolumn{2}{|c|}{$\begin{array}{c}\text { Tetrachloroethene } \\
(\mu \mathrm{g} / \mathrm{L})\end{array}$} & \multicolumn{2}{|c|}{$\begin{array}{l}\text { Trichloroethene } \\
(\mu \mathrm{g} / \mathrm{L})\end{array}$} \\
\hline & $\begin{array}{c}\text { Diffusion } \\
\text { sampler } \\
\text { water }\end{array}$ & $\begin{array}{c}\text { Free- } \\
\text { phase } \\
\text { fuel }\end{array}$ & $\begin{array}{c}\text { Diffusion } \\
\text { sampler } \\
\text { water }\end{array}$ & $\begin{array}{c}\text { Free- } \\
\text { phase } \\
\text { fuel }\end{array}$ & $\begin{array}{c}\text { Diffusion } \\
\text { sampler } \\
\text { water }\end{array}$ & $\begin{array}{l}\text { Free-phase } \\
\text { fuel }\end{array}$ & $\begin{array}{c}\text { Diffusion } \\
\text { sampler } \\
\text { water }\end{array}$ & $\begin{array}{l}\text { Free-phase } \\
\text { fuel }\end{array}$ & $\begin{array}{l}\text { Diffusion } \\
\text { sampler } \\
\text { water }\end{array}$ & $\begin{array}{l}\text { Free-phase } \\
\text { fuel }\end{array}$ \\
\hline $\begin{array}{l}\text { Free product } \\
\text { from well } \\
\text { PW-17 }\end{array}$ & $4 \mathrm{~J}$ & $5,000 \mathrm{U}$ & $5 \mathrm{U}$ & $5,000 \mathrm{U}$ & $3.9 \mathrm{~J}$ & $5,000 \mathrm{U}$ & $5 \mathrm{U}$ & $5,000 \mathrm{U}$ & $2 \mathrm{~J}$ & $5,000 \mathrm{U}$ \\
\hline \multirow{3}{*}{$\begin{array}{l}\text { Free product } \\
\text { from well } \\
\text { MW-11 }\end{array}$} & $10 \mathrm{U}$ & $5,000 \mathrm{U}$ & $10 \mathrm{U}$ & $5,000 \mathrm{U}$ & $10 \mathrm{U}$ & $5,000 \mathrm{U}$ & $7 \mathrm{~J}$ & $4,300 \mathrm{~J}$ & 65 & 5,200 \\
\hline & \multicolumn{2}{|c|}{$\begin{array}{l}\text { Vinyl chloride } \\
(\mu \mathrm{g} / \mathrm{L})\end{array}$} & \multicolumn{2}{|c|}{$\begin{array}{c}\text { Benzene } \\
(\mu \mathrm{g} / \mathrm{L})\end{array}$} & \multicolumn{2}{|c|}{$\begin{array}{l}\text { Ethylbenzene } \\
(\mu \mathrm{g} / \mathrm{L})\end{array}$} & \multicolumn{2}{|c|}{$\begin{array}{c}\text { Toluene } \\
(\mu \mathrm{g} / \mathrm{L})\end{array}$} & \multicolumn{2}{|c|}{$\begin{array}{c}\text { Total xylenes } \\
(\mu \mathrm{g} / \mathrm{L})\end{array}$} \\
\hline & $\begin{array}{c}\text { Diffusion } \\
\text { sampler } \\
\text { wate }\end{array}$ & $\begin{array}{c}\text { Free- } \\
\text { phase } \\
\text { fuel }\end{array}$ & $\begin{array}{c}\text { Diffusion } \\
\text { sampler } \\
\text { water }\end{array}$ & $\begin{array}{c}\text { Free- } \\
\text { phase fuel }\end{array}$ & $\begin{array}{c}\text { Diffusion } \\
\text { sampler } \\
\text { water }\end{array}$ & $\begin{array}{l}\text { Free-phase } \\
\text { fuel }\end{array}$ & $\begin{array}{c}\text { Diffusion } \\
\text { sampler } \\
\text { water }\end{array}$ & $\begin{array}{l}\text { Free-phase } \\
\text { fuel }\end{array}$ & $\begin{array}{l}\text { Diffusion } \\
\text { sampler } \\
\text { water }\end{array}$ & $\begin{array}{l}\text { Free-phase } \\
\text { fuel }\end{array}$ \\
\hline $\begin{array}{l}\text { Free product } \\
\text { from well } \\
\text { PW-17 }\end{array}$ & $5 \mathrm{U}$ & $5,000 \mathrm{U}$ & & $5,000 \mathrm{U}$ & 70 & 21,000 & 112 & $1,100 \mathrm{~J}$ & 350 & 100,000 \\
\hline $\begin{array}{l}\text { Free product } \\
\text { from well } \\
\text { MW-11 }\end{array}$ & $10 \mathrm{U}$ & $5,000 \mathrm{U}$ & $10 \mathrm{U}$ & $5,000 \mathrm{U}$ & 13 & 5,700 & $10 \mathrm{U}$ & $5,000 \mathrm{U}$ & 120 & 43,000 \\
\hline
\end{tabular}

$1,145 \mu \mathrm{g} / \mathrm{L}$ over a vertical distance of about $7 \mathrm{ft}$ (fig. 5). The concentrations decreased with depth at some wells [MW-5 and PW-66 (figs. 5 and 7, respectively)] and increased with depth at others [MW-12 and PW-15 (figs. 2 and 8, respectively)].

The presence of contaminant stratification in well screens has importance for ground-water sampling. In an environment with a sharp concentration gradient, small disturbances in the water column can obscure the stratification. Thus, small amounts of mixing during low-flow sampling can result in large variations in VOC concentrations from pumped samples.

In addition, the potential for stratification is an important consideration when selecting a sampling depth. For example, the data indicate that if the dedicated bladder pump at well MW-12 had been set about $3 \mathrm{ft}$ deeper, the pump would have been in contact with water containing approximately $6,000 \mu \mathrm{g} / \mathrm{L}$ more TCE than was present at the original sampling depth. If the dedicated bladder pump at well MW-5 had been set about $3 \mathrm{ft}$ shallower, the pump would have been in contact with water containing approximately $690 \mu \mathrm{g} / \mathrm{L}$ higher concentrations of TCE. This consideration is even more important for diffusion samplers, which sample only the water in the immediate vicinity of the sampler. Therefore, when using diffusion samplers in a well where chemical stratification is suspected within the screened interval, multiple diffusion samplers can be used to at least initially delineate the stratification. Analytical costs during such an investigation can be minimized by using field gas chromatography to delineate the stratification and to select particular samples for more detailed laboratory analyses.

\section{SUMMARY}

The ground-water VOC concentrations obtained by using water-filled polyethylene diffusion samplers were compared to the ground-water VOC concentrations obtained by using low-flow sampling methods with a peristaltic pump and dedicated bladder pumps in observation wells at Naval Air Station North Island, California. Comparisons of VOC concentrations obtained by using bladder pumps and diffusion samplers showed a generally good correlation. Concentrations of 1,1-dichloroethene (1,1-DCE) and trichloroethene (TCE) in ground water obtained from well MW-9 obtained using the diffusion sampler agreed well (12 and 3 percent difference, respectively) with those samples obtained using the bladder pump. At well MW-5, the TCE concentration in water from the diffusion sampler was higher than in water from the bladder pump, implying that the sample collected by the bladder pump may have underestimated actual concentrations as a result of mixing. Similarly, the 
higher concentrations of vinyl chloride, toluene, and total xylenes in water from the diffusion samplers in wells MW-13A and MW-13B compared to water from the bladder pump imply that the concentrations obtained by the bladder pump may have underestimated actual concentration as a result of mixing in these wells. Concentration differences between the diffusion sampling and bladder-pump sampling methods were noted in samples from well MW-12, and probably are related to mixing in a chemically stratified part of the screened interval. The findings of this investigation suggest that diffusion samplers provide a viable sampling alternative for VOCs in ground water in most tested wells at NAS North Island.

Comparisons of volatile organic compound (VOC) concentrations in water obtained by using diffusion samplers to concentrations obtained by lowflow sampling using a peristaltic pump were used to gain information on the vertical distribution of contamination in the wells. In several wells, the probable effects of mixing or volatization during pumping resulted in lower VOC concentrations in water obtained by using the peristaltic pump compared to concentrations obtained by using the diffusion samplers; however, the data from the low-flow sampling supported the vertical VOC stratification identified by using the diffusion samplers.

Substantial VOC stratification was observed in the screened intervals of several observation wells (MW-12, MW-5, PW-15, and PW-66). The diffusionsampler data show that the vertical change in TCE concentrations over a distance of about $5 \mathrm{ft}$ was approximately $17,500 \mu \mathrm{g} / \mathrm{L}$ in well $\mathrm{PW}-66$, approximately $7,300 \mu \mathrm{g} / \mathrm{L}$ in well PW-15, and approximately $5,900 \mu \mathrm{g} / \mathrm{L}$ in well MW-12. At well MW-5, the 1,1-DCE concentration changed by $3,410 \mu \mathrm{g} / \mathrm{L}$, and the TCE concentration changed by $1,145 \mu \mathrm{g} / \mathrm{L}$ over a vertical distance of about $7 \mathrm{ft}$. Concentrations decreased with depth at some wells (PW-66 and MW-5) and increased with depth at others (MW-12 and PW-15). The presence of stratification in well screens is important for ground-water sampling because small disturbances in the water column can mix the stratification, resulting in large variations in VOC concentrations from pumped samples. The data imply that care must be exercised when selecting a sampling depth. When using diffusion samplers in a well where chemical stratification is suspected within the screened interval, multiple diffusion samplers can be used to at least initially delineate the stratification.
Analytical costs during such an investigation can be minimized by using field gas chromatography or indicator-tube technology to delineate the stratification and to select particular samples for more detailed laboratory analyses.

The diffusion samplers deployed in buckets containing free-phase JP-5 and Stoddard solution collected from observation wells did not show evidence of structural integrity loss during the 2 months of equilibration. The VOCs detected in the free-phase fuel also were detected in water from the diffusion samplers.

\section{REFERENCES}

Gefell, M.J., Hamilton, L.A., and Stout, D.J., 1999, A comparison between low-flow and passive-diffusion bag sampling results for dissolved volatile organics in fractured sedimentary bedrock: Proceedings of the Petroleum and Organic Chemicals in Ground WaterPrevention, Detection, and Remediation Conference, November 17-19, 1999, Houston, Texas, p. 304-315.

OHM Remediation Services Corporation, 2000, Quarterly ground water report, Table 1: Summary of ground water VOC analytical results - UST 653 - December 1999/January 2000: Consultant's Report to U.S. Naval Facilities Command Southwest Division, 9 p.

U.S. Environmental Protection Agency, 1999, On-line SW846 methods: accessed December 21, 1999, at URL tap://search.epa.gov/epaoswer/hazwaste/test/ tx8xxx.htm\#8XXX

Vroblesky, D.A., and Hyde, W.T., 1997, Diffusion samplers as an inexpensive approach to monitoring VOCs in ground water: Ground Water Monitoring and Remediation, v. 17, no. 3, p. 177-184

Vroblesky, D.A., Nietch, C.T., Robertson, J.F., Bradley, P.M., Coates, John, and Morris, J.T., 1999, Natural attenuation potential of chlorinated volatile organic compounds in ground water, TNX flood plain, Savannah River Site, South Carolina: U.S. Geological Survey Water-Resources Investigations Report 99-4071, $43 \mathrm{p}$.

Vroblesky, D.A., Rhodes, L.C., and Robertson, J.F., 1996, Locating VOC contamination in a fractured-rock aquifer at the ground-water/surface-water interface using passive vapor collectors: Ground Water, v. 34, no. 2, p. 223-230.

Vroblesky, D.A., and Robertson, J.F., 1996, Temporal changes in VOC discharge to surface water from a fractured rock aquifer during well installation and operation, Greenville, South Carolina: Ground Water Monitoring and Remediation, v. 16, no. 3, p. 196-201. 
